\title{
ON THE WEIL-PETERSSON VOLUME AND THE FIRST CHERN CLASS OF THE MODULI SPACE OF CALABI-YAU MANIFOLDS
}

\author{
ZHIQIN LU AND XIAOFENG SUN
}

\section{Contents}

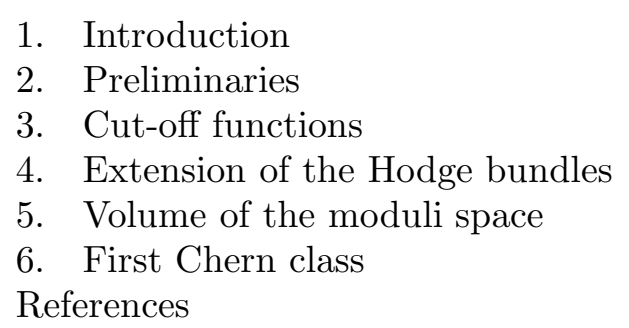

\section{INTRODUCTION}

In this paper, we continue our study of the Weil-Petersson geometry as in the previous paper [10, in which we have proved the boundedness of the Weil-Petersson volume, among the other results. The main results of this paper are that the volume and the integrations of Ricci curvature of the Weil-Petersson metric on the moduli space are rational numbers. In particular, the Ricci curvature defines the first Chern class of the moduli space in the sense of Mumford [11.

It was a classical result of Mumford 11 that for a noncompact Kähler manifold $M$ with $\bar{M}$ being a smooth compactification of $M$ and $\bar{M} \backslash M$ being a divisor $D$ of normal crossings, and for any Hermitian bundle $(E, h)$ over $M$, one can define the Chern classes $c_{k}(E)$ provided the metric $h$ is "good" defined by Mumford [11, Section 1]. Roughly speaking, a metric is "good" if the metric matrix have log bound, and the local connection form and the curvature have Poincaré type growth. It was verified that the natural bundles over locally Hermitian symmetric spaces are "good" (cf. [11]). For the moduli space of curves of genus greater than or equal to 2, the metric induced by the Weil-Petersson metric on the determinant bundle of the log extension of the cotangent bundle is good [19]. However, for the moduli space of polarized Calabi-Yau manifolds, it is not clear that the Weil-Petersson metric or the volume form of the Weil-Petersson metric is "good". By 1, the Weil-Petersson potential is related to the analytic torsion of the moduli space. While the Hessian of the torsion is known to be related to the Weil-Petersson metric and the generalized Hodge metric([3]), it is not easy to find the asymptotic behavior of the BOCV torsion itself. Thus we can not use the theorem of Mumford directly to prove that the integrations are rational numbers.

In this paper, we avoided using the BCOV torsion by the careful analysis of the asymptotic behavior of the Hodge bundles at infinity. Using the Nilpotent Orbit theorem of Schmid [12], we can give another explicit (local) representation of the Weil-Petersson potential. The potential

Date: March 13, 2018.

2000 Mathematics Subject Classification. Primary: 58D27; Secondary: 14J32.

Key words and phrases. Schwarz-Yau lemma, Calabi-Yau manifolds, Weil-Petersson metric.

The first author is supported by NSF Career Award DMS 0347033 and an Alfred P. Sloan Fellowship. The second author is supported by NSF grant DMS 0202508. 
has the following properties: first, as a potential of a Kähler metric, it must be plur-subharmonic. Next, by the Strominger formula, the Ricci curvature of the Weil-Petersson metric is lowerly bounded. Thus the volume form of the Weil-Petersson metric is also plur-subharmonic. Our analysis relies heavily on the above two properties of the potential of the Weil-Petersson metric.

For the noncompact manifold $M$ defined above, we can define a Kähler metric, called the Poincaré metric on $M$ such that on each Euclidean neighborhood of $D$, the metric is asymptotically Poincaré (See $\S\}$ for the precise definition). The metric is not canonically chosen so it doesn't reflect the geometry of the manifold $M$. However, it is complete and the volume is finite, and its curvatures are bounded. In practice, we use the Poincaré metric to bound the other intrinsically defined metrics.

Let $M$ be a Weil-Petersson variety (see $\S 2$ for the definition). It is not clear whether we can construct a Kähler metric with nonpositive sectional curvature on the Weil-Petersson variety. However, if we only require the nonpositivity of the bisectional curvature, then in [8] and [9], the first author defined such a metric, which we called the Hodge metric. In addition to the nonpositivity of the bisectional curvature of the Hodge metric, the holomorphic sectional curvature and the Ricci curvature of Hodge metric are negative and bounded away from zero. By Schwarz-Yau [22] lemma, the Hodge metric is bounded by the Poincaré metric defined above.

Using the comparison of the Hodge and the Poincaré metrics, in the previous paper [10, we have proved that the Weil-Petersson volume and the Hodge volume are all finite. By the definition of the Weil-Petersson metric and the Hodge metric, if a Weil-Petersson variety were compact, then the volume with respect to the Weil-Petersson metric and the Hodge metric could have been rational numbers because of the Gauss-Bonnet Theorem. We thus conjectured that the volume of both metrics are in fact rational numbers, even though the Weil-Petersson varieties are more likely to be noncompact.

In this paper, we verified the conjecture by controlling the growth of the potential of the Weil-Petersson metric at infinity. In order to get the estimates we need, we have to define a special kind of cut-off functions. In general, if a cut-off function is 1 at the origin and if it is supported within a ball of radius $r$, then its second derivatives are of the order $1 / r^{2}$. We can do a little bit better for the Hessian of the cut-off function on $\mathbb{R}^{2}$, because $\mathbb{R}^{2}$ is an example of parabolic manifold defined by P. Li [7. In fact, the order of the Hessian of the cut-off function is of the order $\frac{1}{r^{2}(\log 1 / r)^{2}}$. This observation is important in our proof. By using the cut-off function and the convexity of the Weil-Petersson potential, we can prove

Theorem 1.1. Let $\left(M, \omega_{W P}\right)$ be a Weil-Petersson variety of dimension $m$. Then its volume

$$
\int_{M} \omega_{W P}^{m}
$$

is a rational number.

The volume form of the Weil-Petersson metric has its own convexity by the formula of Strominger. However, in this case, the (local) volume forms are not integrable with respect to the Poincaré metric. Special care must be taken in order to get the similar result as in the volume case. In order to do that, we defined the degeneration order of the volume form along each hypersurface of the divisor $\bar{M} \backslash M$ and then twisted the extension of the anti-canonical bundle of $M$ (See Definition 6.1 for details). Using this, we can prove

Theorem 1.2. Let $X \subset M$ be a subvariety of dimension $q$. Then for non-negative integers $k$ and $l$ with $k+l=q$,

$$
\int_{X}\left(\operatorname{Ric}\left(\omega_{W P}\right)\right)^{k} \wedge \omega_{W P}^{l}
$$

are rational numbers. 
Obviously, Theorem 1.2 implies Theorem 1.1. On the other side, if $X, M$ in Theorem 1.2 are smooth, then we have

Corollary 1.1. Using the above notations, we have

$$
\left(c_{1}\left(\bar{K}_{X}^{-1}\right)+\sum \mu_{i} Y_{i}\right)^{k} \cap c_{1}\left(\bar{F}^{n}\right)^{l}=\int_{X}\left(\operatorname{Ric}\left(\omega_{W P}\right)\right)^{k} \wedge \omega_{W P}^{l},
$$

where $\bar{F}^{n}$ and $\bar{K}_{X}^{-1}$ are the Hodge extensions of the Hodge bundles $F^{n}$ and the anti-canonical bundle $K_{X}^{-1}$ of $X ; \sum Y_{i}=Y$ is the divisor $\bar{X}-X ; Y_{i}$ are irreducible components of $Y$; and $\mu_{i}$ are the degeneration orders of the Weil-Petersson metric along $Y_{i}$.

Remark 1.1. In the above corollary, the righthand side is intrinsically defined. Thus one will get some information of the divisors once the topology of the compact manifold $\bar{X}$ is known. In particular, by using this we can get the information of the monodromy group assuming the moduli space is $C P^{1}$ minus three points. Such a moduli space is of interest in Mirror Symmetry (cf. Doran-Morgan [2]).

Two important papers in the direction of this paper have drawn our attentions. One is the recent survey paper of Todorov [17, which gives a complete summary of the recent progress in the subject. The other one is by Schumacher [13, in which the author computed the curvature of the Weil-Petersson metric of Kähler-Einstein manifolds, using the idea of Siu [14] of horizontal liftings.

We are interested in the volume and the integrations of the curvature because it defines some kind of invariants and using that, we wish to classify polarized Calabi-Yau manifolds and tell the monodromy of the moduli space of Calabi-Yau manifolds ${ }^{1}$. These questions are very important in Mirror Symmetry and we shall study them in a subsequent paper.

The organization of this paper is as follows: in $\S 2$, we give the definition of the Weil-Petersson geometry and some basic estimates; in $\S 3$, we define the Poincaré metric and the cut-off function we need for the rest of the paper; in $\S 4$, we write out the preferred extension of the Hodge bundles defined by the Nilpotent Orbit Theorem explicitly. The main part of the paper is $\S 5$ and $\S 6$, where we prove Theorem 1.1 and Theorem 1.2 .

After finishing this paper, we were informed by A. Todorov that in [16], he proved the rationality of the volume of the moduli space independently.

Acknowledgment. The authors would like to thank P. Li, D. Phong, R. Schoen, and G. Tian for their interest in the work. Particular thanks to K. Liu for his many suggestions and encouragement during the preparation of this paper.

\section{Preliminaries}

In this section, we give the definition of the Weil-Petersson geometry and Weil-Petersson variety, first appeared in [10. Examples of Weil-Petersson varieties are the moduli spaces of polarized Calabi-Yau manifolds.

Definition 2.1. A Weil-Petersson variety is a Kähler orbifold $M$ with the orbifold metric $\omega_{W P}$ such that:

(1) The universal covering space $\widetilde{M}$ is a smooth manifold. There is a natural immersion $\widetilde{M} \rightarrow D$ from $\widetilde{M}$ to the classifying space $D$ (cf. [4]) such that the image of $\widetilde{M}$ is a horizontal slice of $D$. The Hodge bundles $F^{n} \subset \cdots \subset F^{0}$ are defined as the pullback of the tautological bundles of D. Furthermore, we have the natural identification $T M=F^{n-1} / F^{n}$, where $T M$ is the holomorphic tangent bundle of $M$;

\footnotetext{
${ }^{1}$ In fact, our proof strongly hints the relations between the monodromy operators and the rational numbers we defined. In the case that the moduli space is of one-dimensional, an explicit formula can be written down.
} 
(2) $\omega_{W P}$ is the curvature of the bundle $F^{n}$. It is positive definite and thus defines a Kähler metric on $M$ and is called the Weil-Petersson metric;

(3) $M$ is quasi-projective and $F^{n}$ extends to an ample line bundle over the compactification $\bar{M}$ of $M$;

(4) After passing to a finite covering and after desingularization, in a neighborhood of the infinity, $M$ can be written as

$$
\Delta^{n-k} \times\left(\Delta^{*}\right)^{k}
$$

where $\Delta$ is the unit disk and $\Delta^{*}$ is the punctured unit disk. Let $\Omega$ be a local section of $F^{p}$ in the neighborhood, then locally, $\Omega$ can be written as

$$
\Omega=e^{\sqrt{-1}\left(N_{1} \log \frac{1}{z_{1}}+\cdots+N_{k} \log \frac{1}{z_{k}}\right)} A\left(z_{1}, \cdots, z_{n}\right),
$$

where $N_{1}, \cdots, N_{k}$ are nilpotent operators and $A$ is a vector-valued holomorphic function of $z_{1}, \cdots, z_{n}$. Furthermore, all the local sections of all $F^{p}, p=1, \cdots, n$ satisfy the properties in the Nilpotent Orbit theorem of Schmid [12].

A Weil-Petersson subvariety $M_{1}$ is itself a Weil-Petersson variety. Moreover, it is a subvariety of a Weil-Petersson variety $M$ such that if $\bar{M}$ is a compactification of $M$, then $\overline{M_{1}}$, the closure of $M_{1}$ in $\bar{M}$ gives a compactification of $M_{1}$ as a projective variety.

The Weil-Petersson geometry is the geometry of the pair $\left(M, \omega_{W P}\right)$.

Remark 2.1. Moduli spaces of polarized Calabi-Yau manifolds are examples of Weil-Petersson varieties. In fact, for moduli spaces of polarized Calabi-Yau manifolds, the first property of the above definition is the transversality property of Griffiths' [4 variations of Hodge structure. The second property is a theorem of Tian [15] (See also Todorov [18]). The third one is the compactification theorem of Viehweg [20] and the forth property can be verified by the Nilpotent Orbit theorem of Schmid [12.

When we study the boundary behavior of the Weil-Petersson metric on the moduli spaces, we need to analyze the potential $(\Omega, \bar{\Omega})$, where $($,$) is the polarization of the Hodge structures.$ We recall here the result of the potential of Weil-Petersson metric on one dimensional slice of moduli spaces.

Let $\Delta^{*}$ be a one dimensional parameter space of a family of polarized Calabi-Yau manifolds. Let $\Omega$ be a section of the first Hodge bundle $F^{n}$. Then by the Nilpotent Orbit theorem of Schmid [12, after a possible base change, we have

$$
\Omega=e^{\frac{\sqrt{-1}}{2 \pi} N \log \frac{1}{z}} A(z),
$$

where $N$ is the nilpotent operator, $N^{n+1}=0$ for $n$ being the dimension of the Calabi-Yau manifolds, and

$$
A(z)=A_{0}+A_{1} z+\cdots
$$

is a vector-valued convergent power series with the convergent radius $\delta>0$. Let

$$
f_{k, l}(z)=z^{k}\left(\log \frac{1}{z}\right)^{l}
$$

for any $k, l \geq 0$. Then we can write $\Omega$ as the convergent series

$$
\Omega=\sum_{k, l} A_{k, l} z^{k}\left(\log \frac{1}{z}\right)^{l}=\sum_{k, l} A_{k, l} f_{k, l} .
$$

Define $\operatorname{deg} f_{k, l}=k-\frac{l}{n+1}$. Then we have the following lemma (Lemma 7.1 of [10]): 
Lemma 2.1. The convergence of (2.2) is in the $C^{\infty}$ sense. Furthermore, we have

$$
\left\|\Omega-\sum_{\operatorname{deg} f_{k, l} \leq \mu} A_{k, l} f_{k, l}\right\|_{C^{s}} \leq C r^{k_{0}-s}\left(\log \frac{1}{r}\right)^{l_{0}},
$$

where $r=|z|, k_{0}, l_{0}$ are the unique pair of nonnegative integers such that $l_{0} \leq n, k_{0}-\frac{l_{0}}{n+1}>\mu$ and for any pair of integers $k^{\prime}, l^{\prime}$ with $k^{\prime}-\frac{l^{\prime}}{n+1}>\mu$ we have $k^{\prime}-\frac{l^{\prime}}{n+1} \geq k_{0}-\frac{l_{0}}{n+1}$. C is a constant depending only on $k_{0}, l_{0}, \mu$ and $\Omega$.

In order to estimate the volume form and the Chern classes of the Weil-Petersson metric, we also need the following Strominger's formula (Theorem 3.1 of [10]).

Theorem 2.1. Let $\left(g_{i \bar{j}}\right)_{m \times m}$ be the Weil-Petersson metric and let $D_{j} D_{i} \Omega$ be the projection of $\partial_{j} \partial_{i} \Omega$ onto $H^{n-2,2}$. Then the curvature tensor of the Weil-Petersson metric is given by

$$
R_{i \bar{j} k \bar{l}}=g_{i \bar{j}} g_{k \bar{l}}+g_{i \bar{l}} g_{k \bar{j}}-\frac{\left(D_{k} D_{i} \Omega, \overline{\left.D_{l} D_{j} \Omega\right)}\right.}{(\Omega, \bar{\Omega})}
$$

for $1 \leq i, j, k, l \leq m$.

In order to bound the Weil-Petersson metric and its curvature, we need the Hodge metric, which was defined in [8].

Theorem 2.2. Let $D$ be the classifying space. The invariant Hermitian metric of $D$ restricts to $M$ is a Kähler metric called the Hodge metric. Let $\omega_{H}$ be its Kähler form. Then we have

(1) The bisectional curvature of $\omega_{H}$ is nonpositive;

(2) Ric $\left(\omega_{H}\right)<\alpha \omega_{H}<0$ for some negative constant $\alpha$ which only depends on the dimension;

(3) The holomorphic sectional curvature of $\omega_{H}$ is bounded above by $\alpha$;

(4) $2 \omega_{W P} \leq \omega_{H}$ and $-\omega_{H} \leq \operatorname{Ric}\left(\omega_{W P}\right) \leq \omega_{H}$.

The Hodge metric is useful because it gives us the convexity of the volume form of the WeilPetersson metric. Furthermore, using the Hodge metric together with the Schwarz-Yau Lemma, we can control the volume of the Weil-Petersson metric and the Hodge metric.

Theorem 2.3. Let $M$ be the moduli space of polarized Calabi-Yau n-folds. Then the volume of Weil-Petersson subvariety $M_{1}$ of $M$ equipped with the Weil-Petersson metric or the Hodge metric is finite.

\section{Cut-off Functions}

The main result of this section is to prove the existence of the "good" cut-off functions so that our estimates can go through.

We assume that $\bar{M}$ is an $m$-dimensional compact Kähler manifold of dimension $m$ and $D$ be a divisor of $\bar{M}$ with normal crossing so that $M=\bar{M} \backslash D$. We are going to prove that there is a complete Kähler metric on $M$ such that it is asymptotical to the Poincaré metric near infinity. We call this metric the global Poincaré metric or simply the Poincaré metric. We use $\omega_{P}$ to denote its Kähler form.

The result is well known, for example, in [5]. For the sake of completeness and for the setting of notations, we sketch the proof in Lemma 3.1

We let $\bar{M}=U_{1} \cup \cdots \cup U_{t} \cdots \cup U_{s}$ be covered by local coordinate charts where $1 \leq t<s$. Without loss generality, we assume that $\left(\bar{U}_{t+1} \cup \cdots \cup \bar{U}_{s}\right) \cap D=\emptyset$. For each $1 \leq \alpha \leq t$, we assume that there is an $l_{\alpha}$ such that each $U_{\alpha} \backslash D=\left(\Delta^{*}\right)^{l_{\alpha}} \times \Delta^{m-l_{\alpha}}$ and on each $U_{\alpha}, D$ is defined by

$$
z_{1}^{\alpha} \cdots z_{l_{\alpha}}^{\alpha}=0
$$


Let $\left\{\psi_{\alpha}\right\}_{1 \leq \alpha \leq s}$ be the partition of the unity subordinated to the cover $\left\{U_{\alpha}\right\}_{1 \leq \alpha \leq s}$. Let $\omega$ be a Kähler metric of $\bar{M}$ and let $C$ be a large constant. Define

$$
\omega_{P}=C \omega-\sum_{\alpha=1}^{t} \sqrt{-1} \partial \bar{\partial}\left(\psi_{\alpha} \sum_{j=1}^{l_{\alpha}} \log \log \frac{1}{\left|z_{j}^{\alpha}\right|^{2}}\right) .
$$

Then we have

Lemma 3.1. For $C$ large enough, $\omega_{P}$ defines a complete metric on $M$ with finite volume and bounded curvature. Furthermore, there is a constant $C_{1}$ such that

$$
\frac{1}{C_{1}} \omega_{0}^{\alpha} \leq \omega_{P} \leq C_{1} \omega_{0}^{\alpha}
$$

for any $1 \leq \alpha \leq t$, where $\omega_{0}^{\alpha}$ is the local Poincaré metric, defined by

$$
\omega_{0}^{\alpha}=\sum_{i=1}^{l_{\alpha}} \sqrt{-1} \frac{1}{r_{i}^{2}\left(\log \frac{1}{r_{i}}\right)^{2}} d z_{i}^{\alpha} \wedge d \bar{z}_{i}^{\alpha}+\sum_{i=l_{\alpha}+1}^{m} \sqrt{-1} d z_{i}^{\alpha} \wedge d \bar{z}_{i}^{\alpha} .
$$

Proof. This follows from a straightforward computation.

The main result of this section is the following:

Theorem 3.1. Let $\varepsilon>0$. Then there is a function $\rho_{\varepsilon}$ such that

(1) $0 \leq \rho_{\varepsilon} \leq 1$;

(2) For any open neighborhood $V$ of $D$ in $\bar{M}$, there is $\varepsilon>0$ such that $\operatorname{supp}\left(1-\rho_{\varepsilon}\right) \subset V$;

(3) For each $\varepsilon>0$, there is a neighborhood $V_{1}$ of $D$ such that $\left.\rho_{\varepsilon}\right|_{V_{1}} \equiv 0$;

(4) $\rho_{\varepsilon^{\prime}} \geq \rho_{\varepsilon}$ for $\varepsilon^{\prime} \leq \varepsilon$;

(5) There is a constant $C$, independent of $\varepsilon$ such that

$$
-C \omega_{P} \leq \sqrt{-1} \partial \bar{\partial} \rho_{\varepsilon} \leq C \omega_{P}
$$

Proof. The key observation can be explained as follows. For the unit ball in $\mathbb{R}^{n}$, if we construct a smooth function which is 0 on $B_{\varepsilon}(0)$ and 1 outside $B_{2 \varepsilon}(0)$, then the second derivative of the function is in general of order $1 / \varepsilon^{2}$. However, in the two dimensional case, if we consider the Laplacian of the function, then it is possible to lower the order of the second derivative.

Define a decreasing smooth function $\varphi: \mathbb{R} \rightarrow \mathbb{R}, 0 \leq \varphi \leq 1$ as follows:

$$
\varphi(t)= \begin{cases}0 & t \geq 1 \\ 1 & t \leq 0\end{cases}
$$

and assume that $\left|\varphi^{\prime}\right|+\left|\varphi^{\prime \prime}\right|<10$. Define a function $\varphi_{\varepsilon}$ on the unit ball of the complex plane as

$$
\varphi_{\varepsilon}(z)=\varphi\left(\frac{\left(\log \frac{1}{r}\right)^{-1}-\varepsilon}{\varepsilon}\right),
$$

where $r$ is the Euclidean norm of the complex variable $z$. Then we have the following

$$
\begin{aligned}
& \partial_{z} \varphi_{\varepsilon}=\frac{1}{2 \varepsilon} \varphi^{\prime} \frac{1}{z\left(\log \frac{1}{r}\right)^{2}}, \\
& \partial_{z} \bar{\partial}_{z} \varphi_{\varepsilon}=\frac{1}{4 \varepsilon^{2}} \varphi^{\prime \prime} \frac{1}{r^{2}\left(\log \frac{1}{r}\right)^{4}}+\frac{1}{2 \varepsilon} \varphi^{\prime} \frac{1}{r^{2}\left(\log \frac{1}{r}\right)^{3}} .
\end{aligned}
$$

Thus we have 
Lemma 3.2. Using the same notation as above, we have

$$
\begin{gathered}
\left|\partial \varphi_{\varepsilon}\right| \leq \frac{10}{r\left(\log \frac{1}{r}\right)} \\
-10 \sqrt{-1} \frac{1}{r^{2}\left(\log \frac{1}{r}\right)^{2}} d z \wedge d \bar{z} \leq \sqrt{-1} \partial \bar{\partial} \varphi_{\varepsilon} \leq 10 \sqrt{-1} \frac{1}{r^{2}\left(\log \frac{1}{r}\right)^{2}} d z \wedge d \bar{z},
\end{gathered}
$$

where the norm is with respect to the Euclidean metric on $\mathbb{C}$. Furthermore, we have

$$
\operatorname{supp}\left(\partial \varphi_{\varepsilon}\right) \subset B_{e^{-\frac{1}{2 \varepsilon}}}-B_{e^{-\frac{1}{\varepsilon}}}
$$

For $1 \leq \alpha \leq t$ and $\varepsilon>0$ small enough, let

$$
\varphi_{\varepsilon}^{\alpha}\left(z_{1}^{\alpha}, \cdots, z_{m}^{\alpha}\right)=\Pi_{i=1}^{l_{\alpha}}\left(1-\varphi_{\varepsilon}\left(z_{i}^{\alpha}\right)\right)
$$

on $U_{\alpha}$. Then using the Lemma 3.2 , we have

$$
\begin{aligned}
& \left|\partial \varphi_{\varepsilon}^{\alpha}\right| \leq C \sum_{i=1}^{l_{\alpha}}\left|\frac{1}{r_{i}\left(\log \frac{1}{r_{i}}\right)}\right|, \\
& -C \omega_{0}^{\alpha} \leq \sqrt{-1} \partial \bar{\partial} \varphi_{\varepsilon}^{\alpha} \leq C \omega_{0}^{\alpha}
\end{aligned}
$$

for some constant $C$.

We define

$$
\rho_{\varepsilon}=1-\sum_{\alpha}^{t} \psi_{\alpha} \varphi_{\varepsilon}^{\alpha} .
$$

We can verify that $\rho_{\varepsilon}$ satisfies all the properties in Theorem 3.1 by a straightforward computation.

We finish this section by stating the Schwarz-Yau Lemma 22 in our context. The result will be used repeatedly in the rest of this paper:

Proposition 3.1. Let $M$ be a smooth Weil-Petersson variety whose compactification $\bar{M}$ is also smooth. Let $D=\bar{M}-M$ be a divisor of normal crossings. Let $\omega$ is a Kähler metric of $M$ such that its holomorphic sectional curvature is less than a constant $-\delta$ for $\delta>0$. Then there is a constant $C$ such that

$$
\omega \leq C \omega_{P},
$$

where $\omega_{P}$ is the global Poincaré metric.

\section{Extension of the Hodge Bundles}

Let $M$ be a Weil-Petersson variety and $\bar{M}$ be its smooth compactification. We assume that $Y=\bar{M}-M$ be the divisor of normal crossings. Then it is known from [12, page 235] that the Hodge bundles $F^{n}, \cdots, F^{0}$ can be extended to coherent sheaves over $\bar{M}$. Furthermore, if we assume that every element of the monodromy group is unipotent, then the coherent sheaves are in fact vector bundles over $\bar{M}$.

The particular extension of the bundles over $\bar{M}$ is defined by the Nilpotent Orbit theorem of Schmid. In $\S 5$ and $\S 6$, we show that such an extension is the one that we can control the growth of the Weil-Petersson metric and its curvatures at infinity. ${ }^{2}$ Thus although we know the

\footnotetext{
${ }^{2}$ In fact, in the case of the moduli space of Riemann surfaces, there is only one extension of the bundles that is "good" in the sense of Mumford 11] Section 1](cf. [19]). In our case, even if the extension may not be "good", it is the best possible extension we can get.
} 
extension exists by the Nilpotent Orbit theorem, we must write out explicitly the local transition functions.

The first result of this section is the following lemma which is essentially due to Kawamata 6]. We formulate it in the language of Weil-Petersson geometry.

Lemma 4.1. Let $M$ be a Weil-Petersson variety with $\bar{M}$ being its compactification. We assume that $\bar{M}$ is smooth, projective and $Y=\bar{M}-M$ is a divisor of normal crossings. Then there is a divisor $Y_{1}$ of $M$ of normal crossings such that there is a finite covering $M_{1}$ of the variety $M-Y_{1}$ with the following properties:

(1) $M_{1}$ is a Weil-Petersson manifold;

(2) The elements of the monodromy group of $M_{1}$ are unipotent.

Proof. We first observe that if we remove any divisor $Y_{1}$ from $M$, then $M-Y_{1}$ is still a Weil-Petersson variety. For this reason, without loss generality, we can assume that the WeilPetersson variety $M$ is actually a Weil-Petersson manifold.

Now we use the idea of Kawamata [6]. Let $T$ be a monodromy operator which is not unipotent. Let $T=\gamma_{s} \gamma_{u}$ be the decomposition of $T$ into its semi-simple part and its unipotent part. By the theorem of Borel, there is an integer $m$ such that $\gamma_{s}^{m}=1$. Let $L$ be an ample line bundle of $\bar{M}$. Let $Y+Y_{1}=\sum D_{j}$ be the decomposition of the divisor $Y+Y_{1}$ into irreducible pieces, where $Y_{1}$ is the divisor containing the singular locus of $M$. We assume that the monodromy operator $T$ is generated by $U \backslash D_{1}$, where $U$ is a neighborhood of $D_{1}$. Assume that $s$ is large enough such that the bundle $L^{s}\left(-D_{1}\right)$ is very ample. By taking the $m$-th root of the sections of $L^{s}\left(-D_{1}\right)$ we get a variety $M_{1}$ such that outside a possible divisor, it is a finite covering space of M. $M_{1}$ may have some singularities. However, we can always remove those divisors containing singularities to get a smooth manifold. The explicit construction of $M_{1}$ is as follows: let $S_{0}$ be a generic section of the line bundle $L^{s}\left(-D_{1}\right) . S_{0}$ is generic in the sense that on $D_{i}$ with $i \neq 1, S_{0}$ is not identically zero and $d S_{0} \neq 0$ generically on $D_{1}$. We can extend $S_{0}$ to a basis $S_{0}, \cdots, S_{t}$ of $H^{0}\left(L^{s}\left(-D_{1}\right)\right)$ such that the basis defines an embedding

$$
\sigma: \bar{M} \rightarrow \mathbb{C} P^{t}, \quad x \mapsto\left[S_{0}, S_{1}, \cdots, S_{t}\right] .
$$

We consider the map $\pi: C P^{t} \rightarrow C P^{t}$ by $\left[Z_{0}, \cdots, Z_{t}\right] \mapsto\left[Z_{0}^{m}, Z_{1}, \cdots, Z_{t}\right]$. It is a holomorphic $m$-branched covering map. Let $Z$ be the pre-image of $\bar{M}$ under $\pi$. Then $Z$ is a projective variety. Let $Z_{\text {reg }}$ be the smooth points of $Z$. Define $M^{\prime}=Z_{\text {reg }} \cap\left\{Z_{0}=0\right\}$ and let $\tilde{Z}$ be the desingularization of $Z$ along the divisors $Z \backslash M^{\prime}$.

The Hodge bundles can be pulled back to the manifold $M^{\prime}$. At any neighborhood $\left(\Delta^{*}\right)^{l} \times \Delta^{k-l}$ of $\bar{M} \backslash M$, the transform of $(M, \bar{M})$ to $\left(M^{\prime}, \tilde{Z}\right)$ is the $m$-branched covering defined by $z_{1} \mapsto \sqrt[m]{z_{1}}$, where $z_{1}=0$ is corresponding to the divisor $D_{1}$. Evidently, the monodromy operator $T$ is transformed to $T^{m}$, which becomes a unipotent operator.

One can observe that if $T^{\prime}$ is a unipotent monodromy operator, then under the transform $(M, \bar{M}) \mapsto\left(M^{\prime}, \tilde{Z}\right), T^{\prime}$ is still unipotent. Since there are only finitely many irreducible components of $D$, there are only finitely many monodromy operators which are not unipotent. Thus by finitely many transforms, we can get a Weil-Petersson manifold $M_{1}$ on which all monodromy operators are unipotent.

Remark 4.1. In general, a Weil-Petersson variety $M$ may have orbifold singularities. However, we let $M^{\prime}$ be the regular part of $M$. Then $M^{\prime}$ is a manifold that has a smooth compacification $\bar{M}^{\prime}$. By Lemma 4.1. up to a finite covering, we can assume that the elements of the monodromy group are unipotent. Let $M^{\prime \prime} \rightarrow M^{\prime}$ be the finite covering defined by Lemma 4.1, then there is an integer $s$ such that

$$
\operatorname{vol}\left(M^{\prime \prime}\right)=s \operatorname{vol}\left(M^{\prime}\right)
$$


and

$$
\int_{M^{\prime \prime}} c_{1}\left(\omega_{W P}\right)^{k} \wedge \omega_{W P}^{l}=s \int_{M^{\prime}} c_{1}\left(\omega_{W P}\right)^{k} \wedge \omega_{W P}^{l}
$$

Thus From now on, we will prove our results under the additional assumptions that all monodromy operators are unipotent, $M, \bar{M}$ are smooth, and the divisor $Y=\bar{M} \backslash M$ is of normal crossings.

We write out explicitly the extension of the Hodge bundles in terms of the local coordinates.

A bundle $F^{p}$ over $M$ is equivalent to an open cover $\left\{V_{\alpha}\right\}$ of $M$ with transition functions $g_{\alpha \beta}: V_{\alpha} \cap V_{\beta} \rightarrow G L(s, \mathbb{C})$ with $s=\operatorname{rank} F^{p}$. We assume that $\left\{V_{\alpha}\right\}$ is a countable, locally finite cover. Let $\varphi: M \rightarrow \Gamma \backslash D$ be the period map, where $\Gamma$ is the monodromy group. Since $Y \subset \bar{M}$ is compact, we can take a finite cover $\left\{U_{\alpha}\right\}_{\alpha=1}^{t}$ of $Y$ in $\bar{M}$ such that

(1) Each $U_{\alpha}$ is bi-holomorphic to $\Delta^{m}$, the polydisc on $\mathbb{C}^{m}$.

(2) $\bigcup_{1}^{t} U_{\alpha}$ contains $\bar{S}$, where $S$ is a neighbhood of $Y$ in $\bar{M}$.

(3) On each $U_{\alpha}$ with local coordinates $z_{1}^{\alpha}, \cdots, z_{m}^{\alpha}$, the divisor $Y \cap U_{\alpha}$ is given by $z_{1}^{\alpha} \cdots z_{l_{\alpha}}^{\alpha}=0$ for some $l_{\alpha} \in\{1, \cdots, m\}$.

Since $\cup\left\{U_{\alpha}\right\} \cup\left\{V_{\alpha}\right\}$ is an open cover of $\bar{M}$, we can take a finite subcover. After refinement, we can take $V_{1}, \cdots, V_{r} \in\left\{V_{\alpha}\right\}$ such that

(1) $\bigcup_{1}^{t} U_{\alpha} \cup \bigcup_{1}^{r} V_{\alpha}=\bar{M}$.

(2) $\overline{V_{\alpha}} \cap Y=\emptyset$ for $1 \leq \alpha \leq r$.

Now, for each $U_{\alpha}=\Delta^{m}$, let $U_{\alpha}^{*}=U_{\alpha} \backslash Y=\left(\Delta^{*}\right)^{l_{\alpha}} \times \Delta^{m-l_{\alpha}}$. We cut $U_{\alpha}^{*}$ into open conical parts such that each conical part is a product of discs and open sectors with small angles. We write $U_{\alpha}^{*}=\bigcup U_{\alpha, i}$ where each $U_{\alpha, i}$ is a conical domain. ${ }^{3}$

For each $U_{\alpha}^{*}$, the universal covering space of $\widetilde{U}_{\alpha}^{*}$ is $U^{l} \times \Delta^{m-l}$ where $U$ is the upper halfplane and $l=l_{\alpha}$. The natural projection $p: \widetilde{U}_{\alpha}^{*} \rightarrow U_{\alpha}^{*}$ is given by $p\left(w_{1}, \cdots, w_{l}, \cdots, w_{m}\right)=$ $\left(e^{2 \pi i w_{1}}, \cdots, e^{2 \pi i w_{l}}, w_{l+1}, \cdots, w_{m}\right)$. Let $\tilde{M}$ be the universal covering space of $M$. Then we always have the lifting $\tilde{\varphi}: \tilde{M} \rightarrow D$. Locally, it is given by the map $\widetilde{\varphi}_{\alpha}: \widetilde{U}_{\alpha}^{*} \rightarrow D$ corresponding to $\varphi_{\alpha}: U_{\alpha}^{*} \rightarrow \Gamma \backslash D$, and we have the following commutative diagram

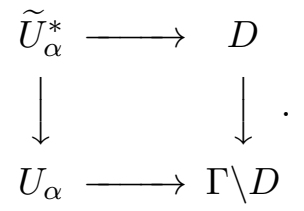

Furthermore, $\tilde{\varphi}_{\alpha}$ and $\tilde{\varphi}_{\beta}$ are compatible if $U_{\alpha} \cap U_{\beta} \neq \emptyset$.

By the Nilpotent Orbit theorem of Schmid, for each $j=1, \cdots, l$, there is a monodromy transform $T_{j}$ such that

$$
\widetilde{\varphi}_{\alpha}\left(w_{1}, \cdots, w_{j}+1, \cdots, w_{m}\right)=T_{j} \circ \widetilde{\varphi}_{\alpha}\left(w_{1}, \cdots, w_{m}\right) .
$$

We have $T_{j} T_{k}=T_{k} T_{j}$ for $1 \leq j, k \leq l$. By Lemma 4.1, we assume that the semisimple part of each $T_{j}$ is 1 . Let

$$
\widetilde{\psi}_{\alpha}\left(w_{1}, \cdots, w_{m}\right)=e^{-\sum_{1}^{l} w_{j} N_{j}} \widetilde{\varphi}_{\alpha}\left(w_{1}, \cdots, w_{m}\right),
$$

where $N_{j}=\log T_{j}$ are the nilpotent operators. Clearly $\widetilde{\psi}_{\alpha}$ is invariant under the transform $w_{j} \mapsto w_{j}+1$ for $j=1, \cdots, l$. So $\widetilde{\psi}_{\alpha}$ descends to a map $\psi_{\alpha}$ from $U_{\alpha}^{*}$ to the complex dual $\check{D}$ of $D$. By the Nilpotent Orbit theorem, $\psi_{\alpha}$ can be holomorphically extended to $U_{\alpha}$.

Since the neighbhood $S$ of $Y$ in $\bar{M}$ satisfies the condition that $\bar{S} \cap V_{\beta}=\emptyset$ for each $V_{\beta}$, and for each $U_{\alpha}$, there is a $\sigma_{\alpha}>0$ such that $\bigcup_{j=1}^{l_{\alpha}} \Delta_{1}^{*} \times \cdots \times \Delta_{j}^{*}\left(\sigma_{\alpha}\right) \times \cdots \times \Delta_{l_{\alpha}}^{*} \times \Delta^{m-l_{\alpha}} \subset S \cap U_{\alpha}$,

\footnotetext{
${ }^{3}$ For example, we can define each $U_{\alpha, i}$ to be $U_{\alpha, i}=\left\{\left(z_{1}^{\alpha}, \cdots, z_{m}^{\alpha}\right) \in U_{\alpha}^{*} \mid \arg z_{i}^{\alpha} \in\left(a_{i}, b_{i}\right), 1 \leq i \leq l_{\alpha}\right\}$, where $\left|b_{i}-a_{i}\right|$ is small.
} 
we know that $\left(\Delta_{1}^{*} \times \cdots \times \Delta_{j}^{*}\left(\sigma_{\alpha}\right) \times \cdots \times \Delta_{l_{\alpha}}^{*} \times \Delta^{m-l_{\alpha}}\right) \cap V_{\beta}=\emptyset$ for any $1 \leq j \leq l_{\alpha}$ and $V_{\beta}$. Let $U_{\alpha, 0}=\left(\Delta^{*}\left(\sigma_{\alpha}\right)\right)^{l_{\alpha}} \times \Delta^{m-l_{\alpha}}$. If $\operatorname{diam} U_{\alpha} \rightarrow 0$, then we can assume that $Y \subset \bigcup U_{\alpha, 0}$ and $U_{\alpha, 0} \cap V_{\beta}=\emptyset$.

Now, $\left\{U_{\alpha, 0}\right\} \cup\left\{U_{\alpha, i}\right\} \cup\left\{V_{\beta}\right\}$ give an open cover of $\bar{M}$. Clearly, on each $U_{\alpha, i}$ and each $V_{\beta}$, the Hodge bundles are trivial. For each $0 \leq p \leq n$, let $s=\operatorname{rank} F^{p}$. We will extend $F^{p}$ to $\bar{M}$ using the cover $\left\{U_{\alpha, 0}\right\} \cup\left\{U_{\alpha, i}\right\} \cup\left\{V_{\beta}\right\}$ of $\bar{M}$. Since $F^{p}$ over each simply connected set of $U_{\alpha}^{*}$ are trivialized by $e^{\frac{\sqrt{-1}}{2 \pi} \sum_{1}^{l} \log \frac{1}{z_{j}} N_{j}} \psi_{\alpha}$, we know that $g_{U_{\alpha, i}, U_{\alpha, j}}=I$ where $I=I_{s}$ is the identity matrix of rank $s$. Since $F^{p}$ is trivial over $U_{\alpha, i}$ and $V_{\beta}$, we know that the transition functions $g_{U_{\alpha, i}, V_{\beta}}$ and $g_{i j}^{\alpha \beta}=g_{U_{\alpha, i}, U_{\beta, j}}$ are given. Since $U_{\alpha, 0} \cap V_{\beta}=\emptyset$, to extend $F^{p}$ to $\bar{M}$, we only need to define $g_{U_{\alpha, 0}, U_{\alpha, j}}, g_{U_{\alpha, 0}, U_{\beta, j}}$, and $g^{\alpha \beta}=g_{U_{\alpha, 0}, U_{\beta, 0}}$.

We define $g_{U_{\alpha, 0}, U_{\alpha, j}}=I$. Since $U_{\alpha, 0} \backslash Y \subset \bigcup U_{\alpha, i}$, we know that for each $q \in U_{\alpha, 0} \cap U_{\beta, j}$, there is a $U_{\alpha, i}$ such that $q \in U_{\alpha, i}$. So naturally we define $g_{U_{\alpha, 0}, U_{\beta, j}}$ on $U_{\alpha, 0} \cap U_{\beta, j} \cap U_{\alpha, i}$ to be the restriction of $g_{U_{\alpha, i}, U_{\beta, j}}$ on $U_{\alpha, 0} \cap U_{\beta, j} \cap U_{\alpha, i}$.

Let $U_{\alpha, i^{\prime}} \cap U_{\alpha, i} \cap U_{\beta, j} \cap U_{\alpha, 0} \neq \emptyset$. Then since $g_{U_{\alpha, i}, U_{\alpha, i^{\prime}}}=I$, we have $g_{U_{\alpha, i}, U_{\beta, j}}=g_{U_{\alpha, i^{\prime}, U_{\beta, j}} \text { on }}$ $U_{\alpha, i^{\prime}} \cap U_{\alpha, i} \cap U_{\beta, j} \cap U_{\alpha, 0}$. Thus $g_{U_{\alpha, 0}, U_{\beta, j}}$ are well-defined.

It is more difficult to define $g^{\alpha \beta}$. For each $q \in U_{\alpha, 0} \cap U_{\beta, 0}$, if $p \notin Y$, then there exist $i$ and $j$ such that $q \in U_{\alpha, i} \cap U_{\beta, j}$. We define $g^{\alpha \beta}(q)=g_{i j}^{\alpha \beta}(q)=g_{U_{\alpha, i}, U_{\beta, j}}$. As long as these transition functions are well-defined, it is trivial to check the compatibility conditions. Thus we need to prove

Claim 1. Using the above notations, we have

(1) $g^{\alpha \beta}$ are well-defined on $U_{\alpha, 0} \cap U_{\beta, 0} \backslash Y$.

(2) $g^{\alpha \beta}$ can be extended to $U_{\alpha, 0} \cap U_{\beta, 0}$.

Remark 4.2. Fixing a pair of charts $U_{\alpha, 0}$ and $U_{\beta, 0}$ such that their intersection is non-empty, we can choose compatible coordinates on $U_{\alpha, 0}$ and $U_{\beta, 0}$. Assume $Y_{\alpha \beta}=U_{\alpha, 0} \cap U_{\beta, 0} \cap Y$ is of codimension $r$ and is given by $z_{1}^{\alpha} \cdots z_{r}^{\alpha}=0$ and $z_{1}^{\beta} \cdots z_{r}^{\beta}=0$ on $U_{\alpha, 0}$ and $U_{\beta, 0}$, respectively. Since these two equations define the same variety $Y_{\alpha \beta}$, we can identify the variables pairwisely in the following way: for each $i=1, \cdots, r$, choose a point $q \in Y_{\alpha \beta}$ such that $z_{i}^{\alpha}(q)=0$ and $z_{j}^{\alpha}(q) \neq 0$ for $j \neq i$. Clearly there is a $t$ with $1 \leq t \leq r$ such that $z_{t}^{\beta}(q)=0$ and $z_{u}^{\beta}(q) \neq 0$ for $u \neq t$. Without loss of generality, we assume that $t=i$. Thus $\frac{z_{i}^{\beta}}{z_{i}^{\alpha}}$ is non-zero for $1 \leq i \leq r$. Furthermore, by slightly shrinking $U_{\alpha, 0}$ and $U_{\beta, 0}$ we can assume that $z_{j}^{\alpha}$ and $z_{j}^{\beta}$ are bounded and are bounded away from 0 on $U_{\alpha, 0} \cap U_{\beta, 0}$ for $r+1 \leq j \leq l_{\alpha}$.

Let $D$ be the classifying space. By definition, this means that $D$ is the space of decompositions of a fixed vector space $H$ satisfying the Hodge-Riemann relations. To prove the first assertion of Claim 1, we fix a $q \in\left(U_{\alpha, 0} \cap U_{\beta, 0}\right) \backslash Y$. Assume that $q \in U_{\alpha, i} \cap U_{\beta, j}$. Let $v_{1}, \cdots, v_{b}$ be a fixed basis of $H$. Using this basis, we can identify a map $\rho$ to the classifying space (or to its compact dual) with a sequence of matrices. Let $\pi_{p} \circ \rho \in F^{p}$. Then $\pi_{p} \circ \rho$ can be represented by a $b \times s$ matrix-valued function, where $s=\operatorname{dim} F^{p}$ as above.

Since $e^{\frac{\sqrt{-1}}{2 \pi} \sum_{1}^{l_{\alpha}} \log \frac{1}{z_{i}^{\alpha}} N_{i}} \psi_{\alpha}$ gives local trivialization of $F^{p}$ over $U_{\alpha, i}$ and $e^{\frac{\sqrt{-1}}{2 \pi} \sum_{1}^{l_{\beta}} \log \frac{1}{z_{j}^{\beta}} N_{j}} \psi_{\beta}$ gives local trivialization of $F^{p}$ over $U_{\beta, j}$, both of them can be represented by $b \times s$ matrices. Fix a lift and lift $\varphi_{\alpha}$ and $\varphi_{\beta}$ to the universal covering spaces $\widetilde{U}_{\alpha}^{*}$ and $\widetilde{U}_{\beta}^{*}$ respectively. We have that, as matrices

$$
\widetilde{\varphi}_{\alpha}\left(w_{1}^{\alpha}, \cdots, w_{m}^{\alpha}\right)=\widetilde{\varphi}_{\beta}\left(w_{1}^{\beta}, \cdots, w_{m}^{\beta}\right) g_{i j}^{\alpha \beta}\left(w_{1}^{\beta}, \cdots, w_{m}^{\beta}\right)
$$


For each $1 \leq i \leq r$, let $T_{i}$ be the monodromy transformation such that

$$
\widetilde{\varphi}_{\alpha}\left(w_{1}^{\alpha}, \cdots, w_{i}^{\alpha}+1, \cdots, w_{m}^{\alpha}\right)=T_{i} \widetilde{\varphi}_{\alpha}\left(w_{1}^{\alpha}, \cdots, w_{i}^{\alpha}, \cdots, w_{m}^{\alpha}\right)
$$

and

$$
\widetilde{\varphi}_{\beta}\left(w_{1}^{\beta}, \cdots, w_{i}^{\beta}+1, \cdots, w_{m}^{\beta}\right)=T_{i} \widetilde{\varphi}_{\beta}\left(w_{1}^{\beta}, \cdots, w_{i}^{\beta}, \cdots, w_{m}^{\beta}\right) .
$$

This is true since $T_{i}$ is corresponding to the same simple loop of $\left(U_{\alpha, 0} \cap U_{\beta, 0}\right) \backslash Y$. Combining the above three formulae, we have

$$
\begin{aligned}
& T_{i} \circ \widetilde{\varphi}_{\beta}\left(w_{1}^{\beta}, \cdots, w_{i}^{\beta}, \cdots, w_{m}^{\beta}\right) g_{i j}^{\alpha \beta}\left(w_{1}^{\beta}, \cdots, w_{i}^{\beta}+1, \cdots, w_{m}^{\beta}\right) \\
= & \widetilde{\varphi}_{\beta}\left(w_{1}^{\beta}, \cdots, w_{i}^{\beta}+1, \cdots, w_{m}^{\beta}\right) g_{i j}^{\alpha \beta}\left(w_{1}^{\beta}, \cdots, w_{i}^{\beta}+1, \cdots, w_{m}^{\beta}\right) \\
= & \widetilde{\varphi}_{\alpha}\left(w_{1}^{\alpha}, \cdots, w_{i}^{\alpha}+1, \cdots, w_{m}^{\alpha}\right) \\
= & T_{i} \circ \widetilde{\varphi}_{\alpha}\left(w_{1}^{\alpha}, \cdots, w_{i}^{\alpha}, \cdots, w_{m}^{\alpha}\right) \\
= & T_{i} \circ \widetilde{\varphi}_{\beta}\left(w_{1}^{\beta}, \cdots, w_{i}^{\beta}, \cdots, w_{m}^{\beta}\right) g_{i j}^{\alpha \beta}\left(w_{1}^{\beta}, \cdots, w_{i}^{\beta}, \cdots, w_{m}^{\beta}\right),
\end{aligned}
$$

which implies

$$
g_{i j}^{\alpha \beta}\left(w_{1}^{\beta}, \cdots, w_{i}^{\beta}+1, \cdots, w_{m}^{\beta}\right)=g_{i j}^{\alpha \beta}\left(w_{1}^{\beta}, \cdots, w_{i}^{\beta}, \cdots, w_{m}^{\beta}\right) .
$$

So $g_{i j}^{\alpha \beta}$ is invariant under the deck transformations of the universal covering of $U_{\alpha, 0} \cap U_{\beta, 0} \backslash Y$ which implies that it descends to a function on $U_{\alpha, 0} \cap U_{\beta, 0} \backslash Y$. This proved that $g_{i j}^{\alpha \beta}$ is welldefined.

Now we prove the second assertion of Claim 11. As stated in the Remark 4.2 if we let $\xi_{i}=\frac{z_{i}^{\beta}}{z_{i}^{\alpha}}$, we know that $\xi_{i}$ is bounded and bounded away from 0 for $1 \leq i \leq r$. Since

$$
e^{\frac{\sqrt{-1}}{2 \pi} \sum_{1}^{l_{\alpha}} \log \frac{1}{z_{i}^{\alpha}} N_{i}} \psi_{\alpha}=e^{\frac{\sqrt{-1}}{2 \pi} \sum_{1}^{l_{\beta}} \log \frac{1}{z_{i}^{\beta}} N_{i}} \psi_{\beta} g_{i j}^{\alpha \beta}
$$

on $U_{\alpha, 0} \cap U_{\beta, 0}$, we have

$$
e^{\frac{\sqrt{-1}}{2 \pi} \sum_{r+1}^{l_{\alpha}} \log \frac{1}{z_{i}^{\alpha}} N_{i}} \psi_{\alpha}=e^{\frac{\sqrt{-1}}{2 \pi}\left(\sum_{1}^{r} \log \frac{1}{\xi_{j}} N_{j}+\sum_{r+1}^{l_{\beta}} \log \frac{1}{z_{i}^{\beta}} N_{i}\right)} \psi_{\beta} g_{i j}^{\alpha \beta} .
$$

By the definition of $\xi_{j}$ and by Remark 4.2 we know that $\log \frac{1}{z_{i}^{\alpha}}$ for $r+1 \leq i \leq l_{\alpha}, \log \frac{1}{z_{i}^{\beta}}$ for $r+1 \leq i \leq l_{\beta}$ and $\log \frac{1}{\xi_{j}}$ for $1 \leq i \leq r$ are bounded. By the Nilpotent Orbit theorem we know that $\psi_{\alpha}$ and $\psi_{\beta}$ can be holomorphically extended to $U_{\alpha, 0}$ and $U_{\beta, 0}$ respectively and image of $\psi_{\alpha}$ and $\psi_{\beta}$ restricted to $Y$ lie in the complex dual of $D$. So we can find a non-singular $r \times r$ minors $A_{\alpha}$ of $\psi_{\alpha}$. Denote the corresponding minor of $\psi_{\beta}$ by $A_{\beta}$. By (4.2), since $N_{i}$ are fixed nilpotent operators, we know that $g_{i j}^{\alpha \beta}=C A_{\alpha} A_{\beta}^{-1}$ where $C=e^{\frac{\sqrt{-1}}{2 \pi} \sum_{r+1}^{l_{\alpha}} \log \frac{1}{z_{i}^{\alpha}} N_{i}-\frac{\sqrt{-1}}{2 \pi} \sum_{1}^{r} \log \frac{1}{\xi_{j}} N_{j}-\frac{\sqrt{-1}}{2 \pi} \sum_{r+1}^{l_{\beta}} \log \frac{1}{z_{i}^{\beta}} N_{i}}$ is inveritible. Since $A_{\beta}$ is also bounded, we know that $g_{i j}^{\alpha \beta}$ is bounded away from 0 . The same argument works for $\left(g_{i j}^{\alpha \beta}\right)^{-1}$. So $g_{i j}^{\alpha \beta}$ is also bounded. This implies that $g_{i j}^{\alpha \beta}$ can be extended to $U_{\alpha, 0} \cap U_{\beta, 0}$ which finishes the construction of the extensions of the Hodge bundles.

Remark 4.3. The tangent bundle of $M$ can be identified with $F^{n-1} / F^{n}$. Since all the Hodge bundles can be extended in the above canonical way, the tangent bundle is also canonically extended. 


\section{Volume of The MOdUli SPACE}

In this section we prove that the volume of the Weil-Petersson metric is a rational number. Let $\Delta_{r}$ be the disk of radius $r$ and $\Delta_{r}^{*}$ be the punctured disk of radius $r$ in $\mathbb{C}$. Let $V_{r}^{k}=\left(\Delta_{r}^{*}\right)^{k} \times \Delta_{r}^{m-k}$ for $1 \leq k \leq m$.

Assume that a chart of $M$ near the boundary is $V_{1}^{k}$ and the Weil-Petersson metric on $V_{1}^{k}$ is defined as $\omega_{W P}=-\sqrt{-1} \partial \bar{\partial} \log (\Omega, \bar{\Omega})$ where $(\Omega, \bar{\Omega})>0$ on $V_{1}^{k}$. We also assume that $\Omega=$ $e^{\frac{\sqrt{-1}}{2 \pi} \sum_{1}^{k} N_{i} \log \frac{1}{z_{i}}} A(z)$ and $\Omega_{0}=e^{\frac{\sqrt{-1}}{2 \pi} \sum_{1}^{k} N_{i} \log \frac{1}{z_{i}}} A_{0}$, where $A_{0}=A(0)$.

The following lemma is one of the key parts in the proof of Theorem 1.1

Lemma 5.1. There exist a universal constant $\delta>0$ which only depends on $\Omega$ such that $\log (\Omega, \bar{\Omega})$ is integrable on $V_{\delta}^{k}$ with respect to the standard Poincaré metric $\omega_{P}$ on $V_{1}^{k}$.

Proof. The proof depends on the convexity of $\log (\Omega, \bar{\Omega})$. Without loss of generality we can assume the convergence radius of $A(z)$ is 1 . Obviously, we have the upper bound

$$
\log (\Omega, \bar{\Omega}) \leq c+\log \Pi_{j=1}^{k}\left(\log \frac{1}{r_{j}}\right)^{n}
$$

where $r_{j}$ is the Euclidean norm of $z_{j}$. Clearly the right hand side of the above formula is integrable with respect to the Poincaré metric $\omega_{P}$ which implies that we only need to check that the integration of $\log (\Omega, \bar{\Omega})$ has a lower bound. Let $\theta_{j}$ be the argument of $z_{j}$ and set

$$
p\left(r_{1}, \cdots, r_{m}\right)=\int_{0}^{2 \pi} \cdots \int_{0}^{2 \pi} \log (\Omega, \bar{\Omega}) d \theta_{1} \cdots d \theta_{m}
$$

Since $-\sqrt{-1} \partial \bar{\partial} \log (\Omega, \bar{\Omega})>0$ we have $\frac{\partial^{2} p}{\partial r_{j}^{2}}+\frac{1}{r_{j}} \frac{\partial p}{\partial r_{j}}<0$ for each $1 \leq j \leq m$ which is equivalent to

$$
\frac{\partial\left(r_{j} \frac{\partial p}{\partial r_{j}}\right)}{\partial r_{j}}<0
$$

In order to prove $\log (\Omega, \bar{\Omega}) \in L^{1}\left(V_{\delta}^{k}, \omega_{P}\right)$ we just need to check that

$$
\int_{0}^{\delta} \cdots \int_{0}^{\delta} p\left(r_{1}, \cdots, r_{m}\right) \frac{r_{k+1} \cdots r_{m}}{r_{1}\left(\log \frac{1}{r_{1}}\right)^{2} \cdots r_{k}\left(\log \frac{1}{r_{k}}\right)^{2}} d r_{1} \cdots d r_{m}>-\infty .
$$

We prove this using mathematical induction on $m$. If the dimension $m$ of the moduli space is 1 , then $k$ must be 1 . By a theorem of Wang [21, we know that the leading term in $(\Omega, \bar{\Omega})$ is $c\left(\log \frac{1}{r_{1}}\right)^{l}$ with $l \geq 1$ if the Weil-Petersson metric is complete at 0 where $c$ is a positive constant. So there is a constant $\delta>0$ such that when $r<\delta,(\Omega, \bar{\Omega}) \geq \frac{c}{2}\left(\log \frac{1}{r_{1}}\right)^{l}$ which implies that $p\left(r_{1}\right) \geq 2 \pi \log \frac{c}{2}+2 \pi l \log \log \frac{1}{r_{1}}$. So

$$
\begin{aligned}
\int_{0}^{\delta} p\left(r_{1}\right) \frac{1}{r_{1}\left(\log \frac{1}{r_{1}}\right)^{2}} d r_{1} & \geq 2 \pi \log \frac{c}{2} \int_{0}^{\delta} \frac{1}{r_{1}\left(\log \frac{1}{r_{1}}\right)^{2}} d r_{1}+2 \pi l \int_{0}^{\delta} \frac{\log \log \frac{1}{r_{1}}}{r_{1}\left(\log \frac{1}{r_{1}}\right)^{2}} d r_{1} \\
& =-\frac{2 \pi \log \frac{c}{2}}{\log \delta}-\frac{2 \pi l}{\log \delta}\left(\log \log \frac{1}{\delta}+1\right)>-\infty
\end{aligned}
$$

If the Weil-Petersson metric is incomplete at 0 , the leading term of $(\Omega, \bar{\Omega})$ is a positive constant $c$. So we can find $\delta>0$ such that $p\left(r_{1}\right) \geq c_{1}$ for some constant $c_{1}$ when $r<\delta$. This implies

$$
\int_{0}^{\delta} p\left(r_{1}\right) \frac{1}{r_{1}\left(\log \frac{1}{r_{1}}\right)^{2}} d r_{1} \geq c_{1} \int_{0}^{\delta} \frac{1}{r_{1}\left(\log \frac{1}{r_{1}}\right)^{2}} d r_{1}=\frac{c_{1}}{\log \frac{1}{\delta}}>-\infty .
$$

Now we assume that when $m \leq s-1$ and $1 \leq k \leq s-1$, the inequality (5.4) hold. Let $m=s$. We first fix $r_{2}, \cdots, r_{m}$. From (5.3) we have $\frac{\partial\left(r_{1} \frac{\partial p}{\partial r_{1}}\right)}{\partial r_{1}}<0$ which implies $r_{1} \frac{\partial p}{\partial r_{1}}$ decreases as $r_{1}$ 
increases. Like the argument above, the leading term of $p$ in $r_{1}$ is either $\log c+l \log \log \frac{1}{r_{1}}$ or $\log c$ where $c$ is a positive function of $r_{2}, \cdots, r_{m}$ and $l \geq 1$ is a positive integer. In either cases we have $\lim _{r_{1} \rightarrow 0} r_{1} \frac{\partial p}{\partial r_{1}}=0$. So we know that $r_{1} \frac{\partial p}{\partial r_{1}}<0$ when $r_{1}>0$ which implies $p$ is decreasing as $r_{1}$ is increasing. So for any $\delta_{1}>0$ small enough, we have

$$
\int_{0}^{\delta_{1}} \frac{p\left(r_{1}, \cdots, r_{m}\right)}{r_{1}\left(\log \frac{1}{r_{1}}\right)^{2}} d r_{1} \geq \int_{0}^{\delta_{1}} \frac{p\left(\delta_{1}, r_{2}, \cdots, r_{m}\right)}{r_{1}\left(\log \frac{1}{r_{1}}\right)^{2}} d r_{1}=-\frac{1}{\log \delta_{1}} p\left(\delta_{1}, r_{2}, \cdots, r_{m}\right) .
$$

Now we go back to $(\Omega, \bar{\Omega})$. We fix a $z_{1}=w \in \Delta^{*}$. Let $\widetilde{\Omega}=\Omega\left(w, z_{2}, \cdots, z_{m}\right)$. Then $-\sqrt{-1} \tilde{\partial} \tilde{\partial} \log (\widetilde{\Omega}, \widetilde{\Omega})$ gives the Weil-Petersson metric on the slice $\tilde{U}=\{w\} \times\left(\Delta^{*}\right)^{k-1} \times \Delta^{m-k}$. If $k>1$, by the induction assumption, we know that $\log (\widetilde{\Omega}, \widetilde{\Omega})$ is integrable on $\tilde{U}$ with respect to the Poincaré metric for some $\delta>0$ and the integration depends on $w$ continuously. This clearly implies

$$
\int_{0}^{2 \pi} \int_{\left(\Delta_{\delta}^{*}\right)^{k-1} \times \Delta_{\delta}^{m-k}} \log (\widetilde{\Omega}, \overline{\widetilde{\Omega}}) \omega_{P}^{m-1} d \theta_{1}>-\infty
$$

Thus we have

$$
\int_{0}^{\delta} \cdots \int_{0}^{\delta}-\frac{1}{\log \delta_{1}} p\left(\delta_{1}, r_{2}, \cdots, r_{m}\right) \frac{r_{k+1} \cdots r_{m}}{r_{1}\left(\log \frac{1}{r_{1}}\right)^{2} \cdots r_{k}\left(\log \frac{1}{r_{k}}\right)^{2}} d r_{2} \cdots d r_{m}>-\infty
$$

So $\log (\Omega, \bar{\Omega})$ is integrable over $V_{\delta}^{k}$ for some $\delta>0$ with respect to the Poincaré metric. If $k=1$, then $\tilde{U}=\{w\} \times \Delta^{m-1}$ and $\log (\widetilde{\Omega}, \widetilde{\Omega})$ is smooth on $\tilde{U}$. Clearly (5.6), (5.7) still hold for some $\delta>0$. So $\log (\Omega, \bar{\Omega})$ is integrable with respect to the Poincaré metric.

Proof of Theorem 1.1. By the Nilpotent Orbit theorem we know that the Hodge bundle $\underline{F}^{n}$ over $M$ can be extended to $\bar{M}$ smoothly. We denoted the extended bundle by $\underline{E}^{n}$. Now we put a Hermitian metric $g$ on $\underline{E}^{n}$. Take an open cover of $\bar{M}$ like we did in 93 , On each $U_{\alpha}$, let $g_{\alpha}$ be a representation of $g$. The local potential of the Weil-Petersson metric on $U_{\alpha}$ is given by $h_{\alpha}=(\Omega, \bar{\Omega})$. Let $f=\frac{h_{\alpha}}{g_{\alpha}}$. It is clear that $f$ is a global function on $M$ although $g_{\alpha}$ and $h_{\alpha}$ are only locally defined. Let $\tilde{\omega}$ be the curvature form of the metric $g$. Then $\omega_{W P}=-\frac{\sqrt{-1}}{2 \pi} \partial \bar{\partial} \log f+\tilde{\omega}$. Since we know that $\tilde{\omega}$ is the first Chern class of the line bundle $\underline{E}^{n}$ over the Kähler manifold $\bar{M}$, we know that

$$
\int_{M} \tilde{\omega}^{m}=\int_{\bar{M}} \tilde{\omega}^{m}
$$

is an integer. We need to prove that $\int_{M} \tilde{\omega}^{m}=\int_{M} \omega_{W P}^{m}$. Using Schwarz-Yau lemma (Proposition [3.1) like we did in [10] we know that $\omega_{W P} \leq c \omega_{P}$ where $\omega_{P}$ is the asymptotic Poincaré metric we constructed in section B. Since $\tilde{\omega}$ is the Ricci form of a line bundle over the compact manifold $\bar{M}$, it is bounded. Thus we can find a constant $c$ such that $-c \omega_{P} \leq \tilde{\omega} \leq c \omega_{P}$.

We check the integrability of $\log f$ on $M$ with respect to the asymptotic metric $\omega_{P}$. Let $\psi_{\alpha}$ be a partition of unity subordinated to the cover $\left\{U_{\alpha}\right\}$. For a chart $U_{\alpha}$ of $\bar{M}$, if $U_{\alpha} \cap \bar{M} \backslash M=\emptyset$ then $\psi_{\alpha} \log f$ is bounded on $U_{\alpha}$. So $\int_{U_{\alpha}} \psi_{\alpha} \log f \omega_{P}^{m}$ is finite. If $U_{\alpha} \cap \bar{M} \backslash M \neq \emptyset$, we know that $\psi_{\alpha} \log f=\psi_{\alpha} \log (\Omega, \bar{\Omega})-\psi_{\alpha} \log g_{\alpha}$. Clearly $\psi_{\alpha} \log g_{\alpha}$ is bounded on $U_{\alpha}$. By Lemma [5.1]we know that $\int_{U_{\alpha}} \psi_{\alpha} \log (\Omega, \bar{\Omega}) \omega_{P}^{m}$ is also finite. This implies $\log f$ is integrable.

Pick an $\varepsilon>0$ small. Let $\rho_{\varepsilon}$ be the cut-off function we constructed in section 3 . We have

$$
\int_{M}\left(\rho_{\varepsilon} \tilde{\omega}^{m}-\rho_{\varepsilon} \omega_{W P}^{m}\right)=\sum_{j=0}^{m-1} \int_{M} \rho_{\varepsilon} \tilde{\omega}^{j} \wedge \omega_{W P}^{m-j-1} \wedge\left(\tilde{\omega}-\omega_{W P}\right) .
$$


For each $0 \leq j \leq m-1$, we have

$$
\begin{aligned}
& \int_{M} \rho_{\varepsilon} \tilde{\omega}^{j} \wedge \omega_{W P}^{m-j-1} \wedge\left(\tilde{\omega}-\omega_{W P}\right)=\int_{M} \rho_{\varepsilon} \tilde{\omega}^{j} \wedge \omega_{W P}^{m-j-1} \wedge \partial \bar{\partial} \log f \\
= & \int_{M} \log f \partial \bar{\partial} \rho_{\varepsilon} \wedge \tilde{\omega}^{j} \wedge \omega_{W P}^{m-j-1}=\int_{\operatorname{supp}\left(1-\rho_{\varepsilon}\right)} \log f \partial \bar{\partial} \rho_{\varepsilon} \wedge \tilde{\omega}^{j} \wedge \omega_{W P}^{m-j-1} \\
= & \sum_{\alpha} \int_{\operatorname{supp}\left(1-\rho_{\varepsilon}\right) \cap U_{\alpha}} \psi_{\alpha} \log f \partial \bar{\partial} \rho_{\varepsilon} \wedge \tilde{\omega}^{j} \wedge \omega_{W P}^{m-j-1}
\end{aligned}
$$

where the sum over $\alpha$ is a finite sum. Also, on each $U_{\alpha}, \log f$ is bounded above by a positive function $c+\sum_{1}^{l_{\alpha}} \log \log \frac{1}{r_{j}}$ which is integrable with respect to the local Poincaré metric. By Theorem 3.1 and the fact that $\tilde{\omega}+\omega_{W P} \leq c \omega_{P}$ (which follows from Proposition 3.1), we know that

$$
\begin{aligned}
& \quad\left|\int_{\operatorname{supp}\left(1-\rho_{\varepsilon}\right) \cap \mathrm{U}_{\alpha}} \psi_{\alpha} \log f \partial \bar{\partial} \rho_{\varepsilon} \wedge \tilde{\omega}^{j} \wedge \omega_{W P}^{m-j-1}\right| \\
& \leq\left|\int_{\operatorname{supp}\left(1-\rho_{\varepsilon}\right) \cap \mathrm{U}_{\alpha}} \psi_{\alpha}\left(\log f+c+\sum_{1}^{l_{\alpha}} \log \log \frac{1}{r_{j}}\right) \partial \bar{\partial} \rho_{\varepsilon} \wedge \tilde{\omega}^{j} \wedge \omega_{W P}^{m-j-1}\right| \\
& \quad+\left|\int_{\operatorname{supp}\left(1-\rho_{\varepsilon}\right) \cap \mathrm{U}_{\alpha}} \psi_{\alpha}\left(c+\sum_{1}^{l_{\alpha}} \log \log \frac{1}{r_{j}}\right) \partial \bar{\partial} \rho_{\varepsilon} \wedge \tilde{\omega}^{j} \wedge \omega_{W P}^{m-j-1}\right| \\
& \leq c_{1}\left|\int_{\operatorname{supp}\left(1-\rho_{\varepsilon}\right) \cap \mathrm{U}_{\alpha}} \psi_{\alpha}\left(\log f+c+\sum_{1}^{l_{\alpha}} \log \log \frac{1}{r_{j}}\right) \omega_{P}^{m}\right| \\
& \quad+c_{1}\left|\int_{\operatorname{supp}\left(1-\rho_{\varepsilon}\right) \cap \mathrm{U}_{\alpha}} \psi_{\alpha}\left(c+\sum_{1}^{l_{\alpha}} \log \log \frac{1}{r_{j}}\right) \omega_{P}^{m}\right|
\end{aligned}
$$

The above expression converges to 0 as $\varepsilon \rightarrow 0$, because $\omega_{P}$ has finite volume and the measure of $\operatorname{supp}\left(1-\rho_{\varepsilon}\right)$ goes to 0 as $\varepsilon \rightarrow 0$ and both $\log f+c+\sum_{1}^{l_{\alpha}} \log \log \frac{1}{r_{j}}$ and $c+\sum_{1}^{l_{\alpha}} \log \log \frac{1}{r_{j}}$ are non-negative and integrable with respect to the metric $\omega_{P}$. Combining (5.8), (5.9) and (5.10) we have

$$
\lim _{\varepsilon \rightarrow 0} \int_{M}\left(\rho_{\varepsilon} \tilde{\omega}^{m}-\rho_{\varepsilon} \omega_{W P}^{m}\right)=0
$$

Thus

$$
\int_{M}\left(\tilde{\omega}^{m}-\omega_{W P}^{m}\right)=\lim _{\varepsilon \rightarrow 0} \int_{M}\left(\rho_{\varepsilon} \tilde{\omega}^{m}-\rho_{\varepsilon} \omega_{W P}^{m}\right)=0 .
$$

This finishes the proof. In general, if the Nilpotent operators $\left\{N_{i}\right\}$ are not unipotent, then by Lemma 4.1] we know that the volume is at least a rational number.

\section{First Chern Class}

Let $M$ be a Weil-Petersson variety of dimension $m$ and let $\omega_{W P}$ be the Kähler form of the Weil-Petersson metric. Like in the previous sections, we let $\bar{M}$ be the compactification of $M$ such that $Y=\bar{M} \backslash M$ is a divisor of normal crossings.

The main result of this section is the following 
Theorem 6.1. Let $\bar{X} \subset \bar{M}$ be a subvariety of dimension $q$. Let $X=\bar{X} \cap M$. Then

$$
\int_{X}\left(\operatorname{Ric}\left(\omega_{W P}\right)\right)^{k} \wedge \omega_{W P}^{l} \in \mathbb{Q}
$$

for $k+l=q$, where the integration is on the smooth part of the variety $X$.

Proof. Without loss generality, we assume that $q=m$ and $X=M$. Let $L_{0}=K_{M}^{-1}$ be the anti-canonical line bundle of $M$. By Lemma 4.1] we assume that all elements of the monodromy group are unipotent. By the remark in $\$$ we know that the tangent bundle of $M$, as a quotient of the Hodge bundles, can be extended to the compactification $\bar{M}$. This implies the $L_{0}$ can be extended to $\overline{L_{0}}$ over $\bar{M}$.

Now let $Y=\bar{M} \backslash M$ be the divisor of $\bar{M}$ of normal crossings. Let $L_{j}$ be the line bundle corresponding to $Y_{j}$ for $j=1, \cdots, p$. We write down the transition functions of $L_{j}$ explicitly. Recall that in Section [4 we constructed an open cover $\left\{U_{\alpha, 0}\right\} \cup\left\{U_{\alpha, i}\right\} \cup\left\{V_{\beta}\right\}$ of $\bar{M}$. Denote this cover by $\mathfrak{U}$. We knew that there is a neighborhood $S$ of $Y$ in $\bar{M}$ such that $\bar{S} \subset \bigcup U_{\alpha, 0}$. Let $\widetilde{U}_{\alpha, j}=U_{\alpha, j} \backslash \bar{S}$. Then $\left\{U_{\alpha, 0}\right\} \cup\left\{\widetilde{U}_{\alpha, i}\right\} \cup\left\{V_{\beta}\right\}$ is also an open cover of $\bar{M}$, denoted by $\widetilde{\mathfrak{U}}$. For each $j=1, \cdots, p$, let $I_{j}$ be the index set such that $\alpha \in I_{j}$ if and only if $U_{\alpha, 0} \cap Y_{j} \neq \emptyset$. From 4 we can assume that for each $\alpha \in I_{j}, U_{\alpha, 0}$ has coordinates $z_{1}^{\alpha}, \cdots, z_{m}^{\alpha}$ and $Y_{j} \cap U_{\alpha, 0}$ is given by $z_{1}^{\alpha}=0$.

Now we define the transition functions. By reordering the elements in $\widetilde{\mathfrak{U}}$, we assume that $\tilde{\mathfrak{U}}=\left\{U_{\alpha}\right\}_{\alpha \in I_{j}} \cup\left\{W_{\gamma}\right\}$, where $U_{\alpha} \cap Y_{j} \neq \emptyset$ and $W_{\gamma} \cap Y_{j}=\emptyset$. When $\alpha, \beta \in I_{j}$ and $U_{\alpha, 0} \cap U_{\beta, 0} \neq \emptyset$, the transition functions are defined to be $g_{U_{\alpha, 0}, U_{\beta, 0}}=\frac{z_{1}^{\alpha}}{z_{1}^{\beta}}$. For $\alpha \in I_{j}$, define $g_{U_{\alpha, 0}, W_{\gamma}}=z_{1}^{\alpha}$ for each $W_{\gamma} \in \tilde{\mathfrak{U}}$ with $U_{\alpha, 0} \cap W_{\gamma} \neq \emptyset$. Finally, for $W_{\gamma}, W_{\delta} \in \tilde{\mathfrak{U}}$ with $W_{\gamma} \cap W_{\delta} \neq \emptyset$, define $g_{W_{\gamma}, W_{\delta}}=1$. One can easily check that these transition functions define the line bundle $L_{j}$.

Now we define the degeneration order of the volume form of the Weil-Petersson metric along each hypersurface $Y_{j}$. We will need the following lemma which is proven in [10]. For completeness, we include the proof here. In the following, we use $\partial=\partial_{z}$ and $\bar{\partial}=\overline{\partial_{z}}$.

Lemma 6.1. Let $f: \mathbb{C} \rightarrow R$ be a degree $k$ homogeneous polynomial of $z$ and $\bar{z}$. Assume that $f(z, \bar{z}) \geq 0$ and is not identically 0 . If there is a constant $c_{1}>0$ such that for every $z$ with $f(z, \bar{z}) \neq 0$, we have

$$
-\frac{c_{1}}{r^{2}\left(\log \frac{1}{r}\right)^{2}} \leq-\partial \bar{\partial} \log f \leq \frac{c_{1}}{r^{2}\left(\log \frac{1}{r}\right)^{2}}
$$

where $r=|z|$, then $k=2 l$ is an even integer and $f(z, \bar{z})=c r^{k}$ where $c$ is a positive constant.

Proof. Let $\theta$ be the argument of $z$. Since $f$ is a homogeneous polynomial which is not identically 0 , we can easily see that the set $\left\{z \in S^{1} \mid f(z, \bar{z})=0\right\}$ is a 0 -dimensional real analytic variety and thus is a discrete set of $S^{1}$. So there are only finite many rays from the origin where $f$ vanishes.

If $k$ is odd, since each term of $f$ has the form $p z^{l} \bar{z}^{k-l}$, we know that

$$
\int_{0}^{2 \pi} f\left(e^{i \theta}, e^{-i \theta}\right) d \theta=0,
$$

which contradicts the fact that $f \geq 0$ and $f\left(e^{i \theta}, e^{-i \theta}\right)$ vanish only for finitely many $\theta$. So $k$ has to be an even integer.

We have

$$
-\partial \bar{\partial} \log f=\frac{\partial f \bar{\partial} f-f \partial \bar{\partial} f}{f^{2}}
$$


Clearly if $\partial f \bar{\partial} f-f \partial \bar{\partial} f$ is not identically 0 , then it is a degree $2 k-2$ homogeneous polynomial. Consequently, $-\partial \bar{\partial} \log f$ is of order $\frac{1}{r^{2}}$ which contradicts to the assumption. Thus we have

$$
\partial f \bar{\partial} f-f \partial \bar{\partial} f=0 .
$$

It is also clear that for a homogeneous polynomial of $z$ and $\bar{z}$, if it is identically 0 , then all of its coefficients are 0 . Now we use induction on $l$. When $l=1$, we have $f=a_{0} z^{2}+a_{1} z \bar{z}+a_{2} \bar{z}^{2}$. From (6.1) we know that $a_{0} a_{1}=a_{0} a_{2}=a_{1} a_{2}=0$. If either $a_{0} \neq 0$ or $a_{2} \neq 0$ we know that the rest of the coefficients are 0 which implies that $f$ is not real. So $a_{0}=a_{2}=0$. Since $f$ is real and non-negative, we know that $a_{1}>0$. The lemma holds. Assuming that the lemma hold when $l \leq p-1$, we consider the case $l=p$. Assume that $f=a_{0} z^{k}+a_{1} z^{k-1} \bar{z}+\cdots+a_{k} \bar{z}^{k}$ where $k=2 p$. If $a_{0} \neq 0$, then we assume $i=\min _{j>0, a_{j} \neq 0} j$. Consider the term $z^{2 k-i-1} \bar{z}^{i-1}$ in $\partial f \bar{\partial} f-f \partial \bar{\partial} f$. We have $k i a_{0} a_{i}-i(k-i) a_{0} a_{i}=0$ which implies $a_{i}=0$ since $a_{0} \neq 0$. This means $f=a_{0} z^{k}$ which contradicts the fact that $f$ is real. So $a_{0}=0$. Similarly we can prove that $a_{k}=0$. So $f=z \bar{z} f_{0}$ where $f_{0}$ is a homogenous polynomial of $z, \bar{z}$ of degree $k-2 . f_{0}$ satisfies the conditions of the lemma. By the assumption, $f_{0}=c z^{p-1} \bar{z}^{p-1}$. So $f=c z^{p} \bar{z}^{p}=c r^{k}$.

Pick a point $q \in Y_{j}$ and assume $q \in U=U_{\alpha, 0}$ with the local coordinates $z=\left(z_{1}, \cdots, z_{m}\right)=$ $z_{\alpha}=\left(z_{1}^{\alpha}, \cdots, z_{m}^{\alpha}\right)$ on $U$. Let $g_{i \bar{j}}$ be the Weil-Petersson metric. In the following, we will use $r_{j}$ and $\theta_{j}$ to denote the Euclidean norm and argument of $z^{j}$. We assume $q \in Y_{j} \backslash \bigcup_{k \neq j} Y_{k}$. Roughly speaking, the degenerate order of $\omega_{W P}^{m}$ along $Y_{j}$ measures the rate of the blow-up or degeneration of the volume form of the Weil-Petersson metric. We need to analyze the asymptotic behavior of $\omega_{W P}^{m}$ when $z \rightarrow q \in Y_{j}$.

Lemma 6.2. Assume $Y_{j} \cap U$ is given by $z_{1}=0$ and $q \in Y_{j} \backslash \bigcup_{k \neq j} Y_{k}$. Then when we expand $(\Omega, \bar{\Omega})^{2 m} \operatorname{det}\left(g_{i \bar{j}}\right)$, the leading term in $z_{1}$ has form $A_{k}\left(z^{\prime}, \overline{z^{\prime}}\right) r_{1}^{k-2}\left(\log \frac{1}{r_{1}^{2}}\right)^{l}$ where $z^{\prime}=\left(z_{2}, \cdots, z_{m}\right)$.

Proof. Near $q$, we know that the local holomorphic section $\Omega$ of the first Hodge bundle $F^{n}$ can be written as $\Omega=e^{\frac{\sqrt{-1}}{2 \pi} N_{1} \log \frac{1}{z_{1}}} A\left(z_{1}, \cdots, z_{m}\right)$ where $A$ is a holomorphic function. Let $z^{\prime}=\left(z_{2}, \cdots, z_{m}\right)$. We can expand $A$ as a power series of $z_{1}, \cdots, z_{m}$ and assume that the convergent radius is 1 . Let $g=\operatorname{det}\left(g_{i \bar{j}}\right)$. For each $i=1, \cdots, m$, let $\Omega_{i}=e^{\frac{\sqrt{-1}}{2 \pi} N_{1} \log \frac{1}{z_{1}}} \partial_{i} A$ and let $\widetilde{\Omega}_{1}=z_{1} \Omega_{1}-\frac{\sqrt{-1}}{2 \pi} N_{1} \Omega$. Let

(1) $\widetilde{g_{11}}=\left(\widetilde{\Omega}_{1}, \bar{\Omega}\right)\left(\Omega, \overline{\widetilde{\Omega}_{1}}\right)-(\Omega, \bar{\Omega})\left(\widetilde{\Omega}_{1}, \overline{\widetilde{\Omega}_{1}}\right)$

(2) $\widetilde{g_{1 j}}=\left(\widetilde{\Omega}_{1}, \bar{\Omega}\right)\left(\Omega, \overline{\Omega_{j}}\right)-(\Omega, \bar{\Omega})\left(\widetilde{\Omega}_{1}, \overline{\Omega_{j}}\right)$ for $2 \leq j \leq m$;

(3) $\widetilde{g_{i \overline{1}}}=\left(\Omega_{i}, \bar{\Omega}\right)\left(\Omega, \widetilde{\Omega_{1}}\right)-(\Omega, \bar{\Omega})\left(\Omega_{i}, \widetilde{\Omega_{1}}\right)$ for $2 \leq i \leq m$;

(4) $\widetilde{g_{i j}}=\left(\Omega_{i}, \bar{\Omega}\right)\left(\Omega, \overline{\Omega_{j}}\right)-(\Omega, \bar{\Omega})\left(\Omega_{i}, \overline{\Omega_{j}}\right)$ for $2 \leq i, j \leq m$.

Let $\widetilde{g}=\operatorname{det}\left(\widetilde{g_{i j}}\right)$. We have $g=\frac{1}{(\Omega, \bar{\Omega})^{2 m}} \frac{1}{r_{1}^{2}} \widetilde{g}$. Since $g>0$ we know that $\widetilde{g}>0$. We expand $A$ with respect to $z_{1}$ so that the coefficient of each term $z_{1}^{s}$ is an analytic function of $z^{\prime}$ and $\overline{z^{\prime}}$. By monodromy theorem, $N_{1}^{n+1}=0$, where $N_{1}$ is the nilpotent operator. Also, since $N_{1}$ is nilpotent, we know that each term in $\widetilde{g}$ has the form $A_{s, t, l} z_{1}^{s} \bar{z}_{1}^{t}\left(\log \frac{1}{r_{1}^{2}}\right)^{l}$ where $A_{s, t, l}=A_{s, t, l}\left(z^{\prime}, \bar{z}^{\prime}\right)$ and $l \leq m n$. Define the degree of such a term by $s+t-\frac{l}{m n+1}$. Assume the lowest degree of terms in the expansion of $\widetilde{g}$ is $k-\frac{l}{m n+1}$. Collect all the terms of degree $k-\frac{l}{m n+1}$ having the form $\sum_{s=0}^{k} A_{s, k-s, l} z_{1}^{s}{\overline{z_{1}}}^{k-s}\left(\log \frac{1}{r_{1}^{2}}\right)^{l}$. Let $f\left(z_{1}, \overline{z_{1}}\right)=\sum_{s=0}^{k} A_{s, k-s, l} z_{1}^{s} \bar{z}_{1}^{k-s}$. We know that, except for a set of lower dimension, $f\left(z_{1}, \overline{z_{1}}\right) \neq 0$ and $f\left(z_{1}, \overline{z_{1}}\right)$ is a homogeneous polynomial of $z_{1}$ and $\overline{z_{1}}$ whose coefficients are functions of $z^{\prime}$ and $\overline{z^{\prime}}$. For each fixed $z^{\prime}$ with $f\left(z_{1}, \overline{z_{1}}\right) \neq 0$, since $f\left(z_{1}, \overline{z_{1}}\right)\left(\log \frac{1}{r_{1}^{2}}\right)^{l}$ is the leading term in the expansion of $\widetilde{g}$, we know that $f\left(z_{1}, \overline{z_{1}}\right) \geq 0$ because 
$\widetilde{g}>0$. We call a point $z^{\prime}$ a generic point in the first direction if for this $z^{\prime}$, we have $f\left(z_{1}, \overline{z_{1}}\right)>0$. For a generic point $z^{\prime}$, we know that $\widetilde{g}=f\left(z_{1}, \overline{z_{1}}\right)\left(\log \frac{1}{r_{1}^{2}}\right)^{l}+g_{0}$ where each term in $g_{0}$ has degree higher than $k-\frac{l}{m n+1}$. Finally we have

$$
\begin{gathered}
-\frac{\sqrt{-1}}{2 \pi} \partial \bar{\partial} \log \widetilde{g}=-\frac{\sqrt{-1}}{2 \pi} \partial \bar{\partial} \log \left(r_{1}^{2}(\Omega, \bar{\Omega})^{2 m} g\right) \\
=-2 m \frac{\sqrt{-1}}{2 \pi} \partial \bar{\partial} \log (\Omega, \bar{\Omega})-\frac{\sqrt{-1}}{2 \pi} \partial \bar{\partial} \log g=2 m \omega_{W P}+\operatorname{Ric}\left(\omega_{W P}\right) .
\end{gathered}
$$

By the Strominger's formula we know that there is a positive constant $c$ such that

$$
-c \omega_{P} \leq \operatorname{Ric}\left(\omega_{W P}\right) \leq c \omega_{P},
$$

where $\omega_{P}$ is the Kähler form of the Poincaré metric. Finally by using Hodge metric and SchwarzYau lemma (Proposition 3.1) we proved in 10] that there is a constant $c$ such that

$$
0<\omega_{W P} \leq c \omega_{P}
$$

Combine all these formulae we know that there is a constant $c$ such that

$$
-c \omega_{P} \leq-\frac{\sqrt{-1}}{2 \pi} \partial \bar{\partial} \log \widetilde{g} \leq c \omega_{P}
$$

which implies

$$
-\frac{c}{r_{1}^{2}\left(\log \frac{1}{r_{1}}\right)^{2}} \leq-\partial_{1} \overline{\partial_{1}} \log \widetilde{g} \leq \frac{c}{r_{1}^{2}\left(\log \frac{1}{r_{1}}\right)^{2}}
$$

Since

$$
-\partial_{1} \overline{\partial_{1}} \log \widetilde{g}=\frac{\partial_{1} \widetilde{g} \overline{\partial_{1}} \widetilde{g}-\widetilde{g} \partial_{1} \overline{\partial_{1}} \widetilde{g}}{\widetilde{g}^{2}}
$$

and the leading term in the numerator is $\left(\partial_{1} f \overline{\partial_{1}} f-f \partial_{1} \overline{\partial_{1}} f\right)\left(\log \frac{1}{r_{1}^{2}}\right)^{2 l}$, the leading term in the denominator is $f^{2}\left(\log \frac{1}{r_{1}^{2}}\right)^{2 l}$, we know that, for generic $z^{\prime}$ we have

$$
-\frac{c}{r_{1}^{2}\left(\log \frac{1}{r_{1}}\right)^{2}} \leq-\partial_{1} \overline{\partial_{1}} \log f \leq \frac{c}{r_{1}^{2}\left(\log \frac{1}{r_{1}}\right)^{2}} .
$$

Using the Lemma 6.1 we have that for generic point $z^{\prime}$ in the first direction, $f\left(z_{1}, \overline{z_{1}}\right)=$ $A_{k}\left(z^{\prime}, \overline{z^{\prime}}\right) r_{1}^{k}$. This implies that, except a lower dimensional set of $z^{\prime}$, the leading term in the expansion of $(\Omega, \bar{\Omega})^{2 m} \operatorname{det}\left(g_{i \bar{j}}\right)$ is $A_{k}\left(z^{\prime}, \overline{z^{\prime}}\right) r_{1}^{k-2}\left(\log \frac{1}{r_{1}^{2}}\right)^{l}$.

Definition 6.1. The degeneration order of $\omega_{W P}^{m}$ along $Y_{j}$ is $\tau_{j}=(k-2) / 2=\mu_{j}$, which is an integer by Lemma 6.1.

Lemma 6.3. The degeneration order is well-defined and is constant except for a lower dimensional set.

Proof. Assume there is another chart $U_{\beta}$ with $U_{\alpha} \cap U_{\beta} \cap Y_{j} \neq \emptyset$. We can also assume that $U_{\beta} \cap$ $Y_{j}$ is given by $z_{1}^{\beta}=0$. From the definition we can see that the degeneration order $\tau_{j}$ is the smallest number $\tau$ such that, for a generic point $z^{\prime}$ in the first direction, $\lim \inf _{z_{1} \rightarrow 0} r_{1}^{\tau}(\Omega, \bar{\Omega})^{2 m} \operatorname{det}\left(g_{i \bar{j}}\right)>$ 0. Since

$$
\omega_{W P}^{m}=\left(\frac{\sqrt{-1}}{2 \pi}\right)^{m} m ! \operatorname{det}\left(\left(g_{\alpha}\right)_{i \bar{j}}\right) d z_{1}^{\alpha} \wedge d \overline{z_{1}^{\alpha}} \cdots d z_{m}^{\alpha} \wedge d \overline{z_{m}^{\alpha}}
$$

is a global form and we can choose the same $\Omega$ on $U_{\alpha} \cap U_{\beta}$, we have

$$
\left(r_{1}^{\alpha}\right)^{\tau} \operatorname{det}\left(\left(g_{\alpha}\right)_{i \bar{j}}\right)=\left.\left.\left(\frac{r_{1}^{\alpha}}{r_{1}^{\beta}}\right)^{\tau}\right|_{17} \operatorname{det}\left(\frac{\partial z_{i}^{\beta}}{\partial z_{j}^{\alpha}}\right)\right|^{2}\left(r_{1}^{\beta}\right)^{\tau} \operatorname{det}\left(\left(g_{\beta}\right)_{i \bar{j}}\right) .
$$


However, we know that $\left(\frac{r_{1}^{\alpha}}{r_{1}^{\beta}}\right)^{\tau}\left|\operatorname{det}\left(\frac{\partial z_{i}^{\alpha}}{\partial z_{j}^{\beta}}\right)\right|^{2}$ is bounded and bounded below from 0 . When we choose $U_{\beta}=U_{\alpha}$ but with a different coordinate system, the above argument implies the degeneration order is independent of the choice of local coordinates. For general $U_{\alpha}$ and $U_{\beta}$, this implies that the order is constant along $Y_{j}$ except for a lower dimensional set.

Now we prove that $\int_{M}\left(\operatorname{Ric}\left(\omega_{W P}\right)\right)^{s} \wedge \omega_{W P}^{m-s} \in \mathbb{Q}$. We first extend the first Hodge bundle $F^{n}$ to the compactification $\bar{M}$ in the way described in 4 . We put a smooth Hermitian metric $h$ on this extended bundle and denote its Kähler form by $\omega$. Recall that we use $L_{0}$ to denote the anti-canonical bundle of $M$ and use $\overline{L_{0}}$ to denote the preferred extension of $L_{0}$ to $\bar{M}$. We put a smooth Hermitian metric $h_{0}$ on $\overline{L_{0}}$. Finally for each line bundle $L_{j}$ corresponding to the hypersurface $Y_{j}$, we put a smooth Hermitian metric $h_{j}$ on it and denote its curvature form by $\omega_{j}$.

We first check that, for all $1 \leq s \leq m, \int_{M}\left(\operatorname{Ric}\left(\omega_{W P}\right)\right)^{s} \wedge \omega_{W P}^{m-s} \in \mathbb{Q}$ is equivalent to

$$
\int_{M}\left(\operatorname{Ric}\left(\omega_{W P}\right)+2 m \omega_{W P}\right)^{s} \wedge \omega_{W P}^{m-s} \in \mathbb{Q}
$$

for all $1 \leq s \leq m$. Clearly we know $\left(\operatorname{Ric}\left(\omega_{W P}\right)+2 m \omega_{W P}\right)^{s} \wedge \omega_{W P}^{m-s}$ is an integer linear combination of terms like $\left(\operatorname{Ric}\left(\omega_{W P}\right)\right)^{i} \wedge \omega_{W P}^{m-i}$ and $\omega_{W P}^{m}$. Also, $\left(\operatorname{Ric}\left(\omega_{W P}\right)\right)^{s} \wedge \omega_{W P}^{m-s}$ is an integer linear combination of terms like $\left(\operatorname{Ric}\left(\omega_{W P}\right)+2 m \omega_{W P}\right)^{j} \wedge \omega_{W P}^{m-j}$ and $\omega_{W P}^{m}$. Since the Weil-Petersson volume is an integer, it is clear that the above argument is true.

The reason we use the expression in (6.2) is that $\operatorname{Ric}\left(\omega_{W P}\right)+2 m \omega_{W P}$ is a nonnegative form.

Realizing that the Ricci curvature of the Weil-Petersson metric is bounded above and below by a constant multiple of the asymptotic Poincaré metric, we can use the same proof in Section 5 to derive that

$$
\int_{M}\left(\operatorname{Ric}\left(\omega_{W P}\right)+2 m \omega_{W P}\right)^{s} \wedge \omega_{W P}^{m-s}=\int_{M}\left(\operatorname{Ric}\left(\omega_{W P}\right)+2 m \omega_{W P}\right)^{s} \wedge \omega_{0}^{m-s}
$$

where $\omega_{0}$ is the curvature form of the line bundle $\bar{L}_{0}$.

Recall that we use $\mu_{j}$ to denote the degeneration order of $\omega_{W P}^{m}$ along the hypersurface $Y_{j}$. Let $\widetilde{\omega}=-\partial \bar{\partial} \log h_{0}+\sum_{j=1}^{p} \mu_{j} \omega_{j}+2 m \omega_{W P}$. Then

$$
\widetilde{\omega}=c_{1}\left(\overline{L_{0}} \oplus L_{1}^{\mu_{1}} \oplus \cdots \oplus L_{p}^{\mu_{p}} \oplus\left(\overline{F^{n}}\right)^{2 m}\right) .
$$

For each line bundle $L_{j}$, on a chart $U_{\alpha}$ at $Y_{j}$, we assume the metric $h_{j}$ is given by $h_{j}^{\alpha}$. From the transition functions that define $L_{j}$ we can see that $h_{j}^{\alpha}\left|z_{1}^{\alpha}\right|^{2}$ is a global function on $\bar{M}$. Denote $f_{j}=\left(h_{j}^{\alpha}\left|z_{1}^{\alpha}\right|^{2}\right)^{\mu_{j}}$. By reordering coordinates, we know that

$$
\widetilde{f}=\frac{\omega_{W P}^{m}}{h_{0} f_{1} \cdots f_{p}}
$$


is a global function on $M$. Also, from 95 if we use $h^{\alpha}$ to denote the smooth Hermitian metric $h$ on $\overline{F^{n}}$ on a chart $U_{\alpha}$, we know that $\bar{f}=\frac{(\Omega, \bar{\Omega})^{2 m}}{\left(h^{\alpha}\right)^{2 m}}$ is also a global function. We have

$$
\begin{aligned}
& \int_{M} \rho_{\varepsilon}\left(\operatorname{Ric}\left(\omega_{W P}\right)+2 m \omega_{W P}\right)^{s} \wedge \omega_{0}^{m-s}-\int_{M} \rho_{\varepsilon} \widetilde{\omega}^{s} \wedge \omega_{0}^{m-s} \\
= & \sum_{j=1}^{s} \int_{M} \rho_{\varepsilon}\left(\operatorname{Ric}\left(\omega_{W P}\right)+2 m \omega_{W P}-\widetilde{\omega}\right) \wedge\left(\operatorname{Ric}\left(\omega_{W P}\right)+2 m \omega_{W P}\right)^{j-1} \wedge \widetilde{\omega}^{s-j} \wedge \omega_{0}^{m-s} \\
= & \sum_{j=1}^{s} \int_{M} \rho_{\varepsilon}\left(-\partial \bar{\partial} \log \widetilde{f}-\partial \bar{\partial} \log f_{1}\right) \wedge\left(\operatorname{Ric}\left(\omega_{W P}\right)+2 m \omega_{W P}\right)^{j-1} \wedge \widetilde{\omega}^{s-j} \wedge \omega_{0}^{m-s} \\
= & \sum_{j=1}^{s} \int_{M} \rho_{\varepsilon}(-\partial \bar{\partial} \log f) \wedge\left(\operatorname{Ric}\left(\omega_{W P}\right)+2 m \omega_{W P}\right)^{j-1} \wedge \widetilde{\omega}^{s-j} \wedge \omega_{0}^{m-s}
\end{aligned}
$$

where $f=\widetilde{f} f_{1}$ is a global positive function on $M$. Integral by part we have

$$
\begin{aligned}
& \int_{M} \rho_{\varepsilon}(\partial \bar{\partial} \log f) \wedge\left(\operatorname{Ric}\left(\omega_{W P}\right)+2 m \omega_{W P}\right)^{j-1} \wedge \widetilde{\omega}^{s-j} \wedge \omega_{0}^{m-s} \\
= & \int_{M} \log f\left(\partial \bar{\partial} \rho_{\varepsilon}\right) \wedge\left(\operatorname{Ric}\left(\omega_{W P}\right)+2 m \omega_{W P}\right)^{j-1} \wedge \widetilde{\omega}^{s-j} \wedge \omega_{0}^{m-s} .
\end{aligned}
$$

Like in the case of $\$ 5$, we need to prove that $\log f$ is locally integrable. We use the same notation as we did in the beginning of $\$ 5$

Consider a chart $U$ at the divisor $Y$. Assume $U=\left(\Delta^{*}\right)^{k} \times \Delta^{m-k}$. From our decomposition $Y=\bigcup Y_{j}$ we can assume that $Y \cap U=\bigcup_{j=1}^{k}\left(Y_{j} \cap U\right)$.

Lemma 6.4. There is a constant $\delta>0$ such that $\log f$ is integrable on $U_{\delta}$ with respect to the standard Poincaré metric on $U$.

Proof. The proof of this lemma is similar to the proof of Lemma 5.1 using convexity. On $U$, we have

$$
\begin{aligned}
f & =\frac{\omega_{W P}^{m}}{h_{0} f_{1} \cdots f_{p}} \frac{(\Omega, \bar{\Omega})^{2 m}}{h^{2 m}}=\frac{\operatorname{det}\left(g_{i \bar{j}}\right)(\Omega, \bar{\Omega})^{2 m}}{h_{0}\left(h_{1}\left|r_{1}\right|^{2}\right)^{\mu_{1}} \cdots\left(h_{k}\left|r_{k}\right|^{2}\right)^{\mu_{k}}} \frac{1}{f_{k+1} \cdots f_{p} h^{2 m}} \\
& =g_{0} \frac{1}{h_{0} h_{1}^{\mu_{1}} \cdots h_{k}^{\mu_{k}} f_{k+1} \cdots f_{p} h^{2 m}}=g_{0} g_{1},
\end{aligned}
$$

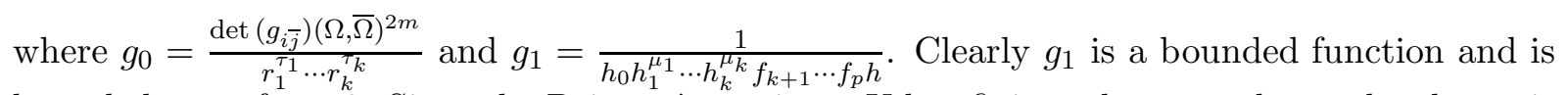
bounded away from 0 . Since the Poincaré metric on $U$ has finite volume, we know that $\log g_{1}$ is locally integrable with respect to the Poincaré metric. Since

$$
-\frac{\sqrt{-1}}{2 \pi} \partial \bar{\partial} \log f=\operatorname{Ric}\left(\omega_{W P}\right)+2 m \omega_{W P}-\widetilde{\omega} \geq-c \omega_{P}
$$

and $-\partial \bar{\partial} \log g_{1}$ is bounded we have

$$
-\frac{\sqrt{-1}}{2 \pi} \partial \bar{\partial} \log g_{0}=-\frac{\sqrt{-1}}{2 \pi} \partial \bar{\partial} \log f+\frac{\sqrt{-1}}{2 \pi} \partial \bar{\partial} \log g_{1} \geq-c \omega_{P}
$$

From the definition of the degeneration order we know that the leading term of $g_{0}$ with respect to $z_{j}$ for $1 \leq j \leq k$ is $A_{j}\left(z^{\prime}, \overline{z^{\prime}}\right)\left(\log \frac{1}{r_{j}^{2}}\right)^{l_{j}}$ with $l_{j} \leq m n$ and $z^{\prime}=\left(z_{1}, \cdots, z_{j-1}, z_{j+1}, \cdots, z_{m}\right)$. This implies that $g_{0} \leq c\left(\prod_{1}^{k} \log \frac{1}{r_{j}^{2}}\right)^{m n}$ which is integrable with respect to the Poincaré metric. 
So we only need to prove that

$$
\int_{U} \log g_{0} \omega_{P}^{m}>-\infty
$$

Set

$$
p\left(r_{1}, \cdots, r_{m}\right)=\int_{0}^{2 \pi} \cdots \int_{0}^{2 \pi} \log g_{0} d \theta_{1} \cdots d \theta_{m} .
$$

From (6.9) we can easily see that for $1 \leq j \leq k$,

$$
\frac{\partial\left(r_{j} \frac{\partial p}{\partial r_{j}}\right)}{\partial r_{j}} \leq \frac{c}{r_{j}\left(\log \frac{1}{r_{j}}\right)^{2}}
$$

Now, for generic $z^{\prime}$, since the leading term of $g_{0}$ is $A_{j}\left(z^{\prime}, \overline{z^{\prime}}\right)\left(\log \frac{1}{r_{j}^{2}}\right)^{l_{j}}$ where $A_{j}\left(z^{\prime}, \overline{z^{\prime}}\right)>0$, we know that

$$
\lim _{r_{j} \rightarrow 0} r_{j} \frac{\partial p}{\partial r_{j}}=0
$$

Now integrate both sides of (6.12) from 0 to $r_{j}$, using (6.13), we have

$$
r_{j} \frac{\partial p}{\partial r_{j}} \leq \frac{c}{\log \frac{1}{r_{j}}}
$$

Fix a $\delta>0$ to be chosen, we have that, for generic $z^{\prime}$

$$
\begin{aligned}
& p\left(r_{1}, \cdots, r_{j-1}, \delta, r_{j+1}, \cdots, r_{m}\right)-p\left(r_{1}, \cdots, r_{m}\right) \\
\leq & \int_{r_{j}}^{\delta} \frac{c}{r_{j} \log \frac{1}{r_{j}}} d r_{j}=\log \log \frac{1}{r_{j}}-\log \log \frac{1}{\delta}
\end{aligned}
$$

which implies

$$
p\left(r_{1}, \cdots, r_{m}\right) \geq p\left(r_{1}, \cdots, r_{j-1}, \delta, r_{j+1}, \cdots, r_{m}\right)+\log \log \frac{1}{\delta}-\log \log \frac{1}{r_{j}} .
$$

From (6.10) we only need to show that

$$
\int_{U} \log g_{0} \omega_{P}^{m}=\int_{0}^{\delta} \cdots \int_{0}^{\delta} p\left(r_{1}, \cdots, r_{m}\right) \frac{r_{k+1} \cdots r_{m}}{r_{1}\left(\log \frac{1}{r_{1}}\right)^{2} \cdots r_{k}\left(\log \frac{1}{r_{k}}\right)^{2}} d r_{1} \cdots d r_{m}>-\infty .
$$

We prove (6.16) using induction on $k$. If $k=0$, then $p$ is bounded which implies (6.16) is true. Assume that for $k \leq l$ the argument is true, consider $k=l+1$. From (6.15) we know that for generic $z^{\prime}=\left(z_{2}, \cdots, z_{m}\right)$, we have

$$
\begin{aligned}
& \int_{0}^{\delta} p\left(r_{1}, \cdots, r_{m}\right) \frac{r_{k+1} \cdots r_{m}}{r_{1}\left(\log \frac{1}{r_{1}}\right)^{2} \cdots r_{k}\left(\log \frac{1}{r_{k}}\right)^{2}} d r_{1} \\
\geq & \int_{0}^{\delta} p\left(\delta, r_{2}, \cdots, r_{m}\right) \frac{r_{k+1} \cdots r_{m}}{r_{1}\left(\log \frac{1}{r_{1}}\right)^{2} \cdots r_{k}\left(\log \frac{1}{r_{k}}\right)^{2}} d r_{1} \\
& +\int_{0}^{\delta}\left(\log \log \frac{1}{\delta}-\log \log \frac{1}{r_{1}}\right) \frac{r_{k+1} \cdots r_{m}}{r_{1}\left(\log \frac{1}{r_{1}}\right)^{2} \cdots r_{k}\left(\log \frac{1}{r_{k}}\right)^{2}} d r_{1} .
\end{aligned}
$$

The second term in the above formula is integrable with respect to $r_{2}, \cdots, r_{m}$ on $[0, \delta]^{m-1}$ by direct computation. To estimate the first term, we can choose a generic $\delta$ and working on the lower dimensional piece $\left\{\left|z_{1}\right|=\delta\right\} \cap U$. By induction assumption, the first term is also integrable with respect to $r_{2}, \cdots, r_{m}$ on $[0, \delta]^{m-1}$. This finishes the proof. 
Now we go back to the proof of the main theorem. From the above lemma we know that $\log f$ is integrable with respect to the Poincaré metric on each chart that touches the divisor $Y$. On those charts which do not touch $Y, \log f$ is bounded. Using a partition of unity, we can easily see that $\log f \in L^{1}\left(M, \omega_{P}\right)$ where $\omega_{P}$ is the (global) asymptotic Poincaré metric on $M$. From (6.7) we have

$$
\begin{aligned}
& \int_{M} \log f\left(\frac{\sqrt{-1}}{2 \pi} \partial \bar{\partial} \rho_{\varepsilon}\right) \wedge\left(\operatorname{Ric}\left(\omega_{W P}\right)+2 m \omega_{W P}\right)^{j-1} \wedge \widetilde{\omega}^{s-j} \wedge \omega_{0}^{m-s} \\
= & \int_{\operatorname{supp}\left(1-\rho_{\varepsilon}\right)} \log f\left(\frac{\sqrt{-1}}{2 \pi} \partial \bar{\partial} \rho_{\varepsilon}\right) \wedge\left(\operatorname{Ric}\left(\omega_{W P}\right)+2 m \omega_{W P}\right)^{j-1} \wedge \widetilde{\omega}^{s-j} \wedge \omega_{0}^{m-s} \\
= & \sum_{\alpha} \int_{\operatorname{supp}\left(1-\rho_{\varepsilon}\right) \cap U_{\alpha}} \psi_{\alpha} \log f\left(\frac{\sqrt{-1}}{2 \pi} \partial \bar{\partial} \rho_{\varepsilon}\right) \wedge\left(\operatorname{Ric}\left(\omega_{W P}\right)+2 m \omega_{W P}\right)^{j-1} \wedge \widetilde{\omega}^{s-j} \wedge \omega_{0}^{m-s}
\end{aligned}
$$

where the sum over $\alpha$ is a finite sum. Since on each $U_{\alpha}, \log f$ is bounded above by $c \sum_{1}^{l_{\alpha}} \log \log \frac{1}{r_{j}}$ and both $\operatorname{Ric}\left(\omega_{W P}\right)+2 m \omega_{W P}$ and $\widetilde{\omega}$ are bounded above and below by a constant multiple of the Poincaré metric, using the same technique as in (5.10), since the measure of $\operatorname{supp}\left(1-\rho_{\varepsilon}\right)$ goes to 0 as $\varepsilon \rightarrow 0$, we conclude that

$$
\int_{M} \log f\left(\frac{\sqrt{-1}}{2 \pi} \partial \bar{\partial} \rho_{\varepsilon}\right) \wedge\left(\operatorname{Ric}\left(\omega_{W P}\right)+2 m \omega_{W P}\right)^{j-1} \wedge \widetilde{\omega}^{s-j} \wedge \omega_{0}^{m-s}=0
$$

which implies

$$
\int_{M} \rho_{\varepsilon}\left(\operatorname{Ric}\left(\omega_{W P}\right)+2 m \omega_{W P}\right)^{s} \wedge \omega_{0}^{m-s}=\int_{M} \rho_{\varepsilon} \widetilde{\omega}^{s} \wedge \omega_{0}^{m-s}
$$

Again, since $-c \omega_{P} \leq \operatorname{Ric}\left(\omega_{W P}\right)+2 m \omega_{W P} \leq c \omega_{P}$ and $-c \omega_{P} \leq \widetilde{\omega} \leq c \omega_{P}$, by the dominate convergence theorem, let $\varepsilon \rightarrow 0$ in (6.19) we have

$$
\int_{M}\left(\operatorname{Ric}\left(\omega_{W P}\right)+2 m \omega_{W P}\right)^{s} \wedge \omega_{0}^{m-s}=\int_{M} \widetilde{\omega}^{s} \wedge \omega_{0}^{m-s}
$$

Since

$$
\int_{M} \widetilde{\omega}^{s} \wedge \omega_{0}^{m-s}=\int_{\bar{M}} \widetilde{\omega}^{s} \wedge \omega_{0}^{m-s}
$$

and $\widetilde{\omega}^{s} \wedge \omega_{0}^{m-s}$ is a characteristic class on $\bar{M}$, we know that $\int_{M} \widetilde{\omega}^{s} \wedge \omega_{0}^{m-s} \in \mathbb{Z}$ which implies that $\int_{M}\left(\operatorname{Ric}\left(\omega_{W P}\right)\right)^{s} \wedge \omega_{W P}^{m-s} \in \mathbb{Z}$, if the nilpotent operators are unipotent. In general, by Lemma 4.1] the integral is a rational number.

We end this paper by the obvious possible generalization of this paper:

Conjecture. Let $c_{k}\left(\omega_{W P}\right)$ be the $k$-th elementary polynomial of the curvature tensor of the Weil-Petersson metric. Let $X$ be any Weil-Petersson subvariety of dimension $q$ of a WeilPetersson variety $M$. Then

$$
\int_{X} c_{k}\left(\omega_{W P}\right) \wedge \omega_{W P}^{l}
$$

is a rational number, where $k+l=q$.

It would be interesting to see if this is true in the category of Weil-Petersson geometry. 


\section{REFERENCES}

[1] M. Bershadsky, S. Cecotti, H. Ooguri, and C. Vafa. Kodaira-Spencer theory of gravity and exact results for quantum string amplitudes. Comm. Math. Phys., 165(2):311-427, 1994.

[2] C. Doran and J. Morgan. Integral monodromy and Calabi-Yau Moduli. preprint.

[3] H. Fang and Z. Lu. Generalized Hodge metrics and BCOV torsion on Calabi-Yau moduli. to appear at Journal fur die reine und angewandte Mathematik, 2003.

[4] P. Griffiths, editor. Topics in transcendental algebraic geometry, volume 106 of Annals of Mathematics Studies, Princeton, NJ, 1984. Princeton University Press.

[5] J. Jost and S.-T. Yau. Harmonic mappings and algebraic varieties over function fields. Amer. J. Math., 115(6):1197-1227, 1993.

[6] Y. Kawamata. Characterization of abelian varieties. Compositio Math., 43(2):253-276, 1981.

[7] P. Li. Curvature and function theory on Riemannian manifolds. In Surveys in differential geometry, Surv. Differ. Geom., VII, pages 375-432. Int. Press, Somerville, MA, 2000.

[8] Z. Lu. On the geometry of classifying spaces and horizontal slices. Amer. J. Math., 121(1):177-198, 1999.

[9] Z. Lu. On the Hodge metric of the universal deformation space of Calabi-Yau threefolds. J. Geom. Anal., 11(1):103-118, 2001.

[10] Z. Lu and X. Sun. Weil-Petersson geometry on moduli space of polarized Calabi-Yau manifolds. J. Inst. Math. Jussieu, 3(2):185-229, 2004.

[11] D. Mumford. Hirzebruch's proportionality theorem in the noncompact case. Invent. Math., 42:239-272, 1977.

[12] W. Schmid. Variation of Hodge structure: the singularities of the period mapping. Invent. Math., 22:211-319, 1973.

[13] G. Schumacher. The curvature of the Petersson-Weil metric on the moduli space of Kähler-Einstein manifolds. In Complex analysis and geometry, Univ. Ser. Math., pages 339-354. Plenum, New York, 1993.

[14] Y. T. Siu. Curvature of the Weil-Petersson metric in the moduli space of compact Kähler-Einstein manifolds of negative first Chern class. In Contributions to several complex variables, Aspects Math., E9, pages 261-298. Vieweg, Braunschweig, 1986.

[15] G. Tian. Smoothness of the universal deformation space of compact Calabi-Yau manifolds and its PeterssonWeil metric. In Mathematical aspects of string theory (San Diego, Calif., 1986), volume 1 of Adv. Ser. Math. Phys., pages 629-646. World Sci. Publishing, Singapore, 1987.

[16] A. Todorov. Weil-petersson volumes of the moduli spaces of cy manifolds. hep-th/0408033.

[17] A. N. Todorov. Introduction to Weil-Petersson Geometry of the moduli space of CY manifolds. preprint.

[18] A. N. Todorov. The Weil-Petersson geometry of the moduli space of $\mathrm{SU}(n \geq 3)$ (Calabi-Yau) manifolds. I. Comm. Math. Phys., 126(2):325-346, 1989.

[19] S. Trapani. On the determinant of the bundle of meromorphic quadratic differentials on the Deligne-Mumford compactification of the moduli space of Riemann surfaces. Math. Ann., 293(4):681-705, 1992.

[20] E. Viehweg. Quasi-projective moduli for polarized manifolds, volume 30 of Ergebnisse der Mathematik und ihrer Grenzgebiete (3) [Results in Mathematics and Related Areas (3)]. Springer-Verlag, Berlin, 1995.

[21] C.-L. Wang. On the incompleteness of the Weil-Petersson metric along degenerations of Calabi-Yau manifolds. Math. Res. Lett., 4(1):157-171, 1997.

[22] S. T. Yau. A general Schwarz lemma for Kähler manifolds. Amer. J. Math., 100(1):197-203, 1978.

(Zhiqin Lu and Xiaofeng Sun) Department of Mathematics, University of California, Irvine, Irvine, CA 92697

E-mail address, Zhiqin Lu: zlu@math.uci.edu

E-mail address, Xiaofeng Sun: xsun@math.uci.edu 